\title{
Temporal regulation of prenatal embryonic development by paternal
}

\section{imprinted loci}

Qing Li, ${ }^{1,3}$ Yuanyuan Li, ${ }^{1,3}$ Qi Yin, ${ }^{1,3}$ Shuo Huang, ${ }^{2,3}$ Kai Wang,,${ }^{1,3}$ Liangchai Zhuo, ${ }^{2}$ Wei Li, ${ }^{1}$ Boran Chang ${ }^{1}$ and Jinsong $\mathrm{Li}^{1,2, *}$

${ }^{1}$ State Key Laboratory of Cell Biology, CAS Center for Excellence in Molecular Cell Science, Shanghai Key Laboratory of Molecular Andrology, Shanghai Institute of Biochemistry and Cell

Biology, Chinese Academy of Sciences, University of Chinese Academy of Sciences, 20031

Shanghai, China

${ }^{2}$ School of Life Science and Technology, Shanghai Tech University, 20031 Shanghai, China.

${ }^{3}$ These authors contributed equally to the work

*Correspondence: jsli@ sibcb.ac.cn (J. L.) 


\begin{abstract}
$H 19$ and $G t l 2$ are paternal imprinted genes that are pivotal for prenatal embryonic development. Meanwhile, mouse nongrowing oocytes and sperm- or oocyteoriginated haploid embryonic stem cells (haESCs) carrying both $H 19$ and $I G$-DMR (differentially DNA-methylated region) deletions (DKO) that partially mimic paternal imprinting of H19-Igf2 and Dlk1-Dio3 can be employed as sperm replacement to efficiently support full-term embryonic development. However, how H19-DMR and $I G$-DMR act together to regulate embryonic development is still largely unknown. Here, using androgenetic haESC (AG-haESC)-mediated semi-cloned (SC) technology, we showed that paternal H19-DMR and $I G$-DMR are not essential for pre-implantation development of SC embryos generated through injection of AGhaESCs into oocytes. H19-DMR plays critical roles before 12.5 days of gestation while $I G$-DMR is essential for late-gestation of SC embryos. Interestingly, we found that combined deletions of $H 19$ and H19-DMR can further improve the efficiency of normal development of SC embryos at mid-gestation compared to DKO SC embryos. Transcriptome and histology analyses revealed that $H 19$ and H19-DMR combined deletions rescue the placental defects. Furthermore, we showed that H19, H19-DMR and $I G$-DMR deletions (TKO) give rise to better prenatal and postnatal embryonic development of SC embryos compared to DKO. Together, our results indicate the temporal regulation of paternal imprinted loci during embryonic development.
\end{abstract}

Imprinted loci, semi-cloned technology, temporal regulation, H19-Igf2, Dlk1Dio3, embryonic development 


\section{INTRODUCTION}

In the 1970s, many groups attempted to induce mouse parthenogenetic (PG) development, however, the PG embryos could not survive after 10 days of gestation (Graham, 1970; Kaufman, 1973; Tarkowski, 1975). In 1984, two elegant embryological experiments by construction of mouse embryos carrying only paternal or maternal genome provided direct evidence to demonstrate nonequivalence of parental genome, the concept of genomic imprinting (McGrath and Solter, 1984; Surani et al., 1984). Genomic imprinting, an epigenetic mechanism that results in parent-of-origin specific gene expression, plays essential roles in the regulation of mammalian embryonic and extraembryonic development (Barlow and Bartolomei, 2014; Ferguson-Smith, 2011).

Among the 151 reported murine imprinted genes (http://www.mousebook.org/imprinting-genelist), the H19-Igf 2 locus, the first identified imprinted region, is critical for normal prenatal growth and placentation and regulated by differentially DNA methylation (H19-DMR) (Arney, 2003; Bartolomei et al., 1991; Cleaton et al., 2014; DeChiara et al., 1991). H19-DMR, located 2 to $4 \mathrm{~kb}$ upstream from the start of transcription start site (Thorvaldsen et al., 1998; Tremblay et al., 1997), is essential for establishing the pattern of imprinting by which H19, a non-coding RNA, is exclusively expressed from the maternal chromosome (Bartolomei et al., 1991) and Igf2 is only active paternally (DeChiara et al., 1991). On the maternal chromosome, the DMR is hypomethylated and bound by the zinc-finger protein CCCTC-binding factor (CTCF) (Bell et al., 1999), leading to the establishment of an insulator act as a barrier to influence the neighboring cisacting elements and silence $\operatorname{Ig} f 2$ on the maternal allele (Bell and Felsenfeld, 2000; Hark et al., 2000). On the paternal chromosome, the DMR is hypermethylated and not bound by CTCF, thus allowing downstream enhancers to activate paternal $\lg 2$ gene (Bell and Felsenfeld, 2000; Hark et al., 2000). H19 knock-out mice are viable, fertile, but present an overgrowth phenotype (Leighton et al., 1995). In contrast, overexpression of $H 19$ was deleterious to embryo development after embryonic day 14 (E14) (Brunkow and Tilghman, 1991). Deletion of Igf2 leads to fetal and placental growth restriction (Constância et al., 2002; DeChiara et al., 1990), whereas overexpression of the $\operatorname{Ig} f 2$ by imprint relaxation leads to placental and fetal overgrowth (Eggenschwiler et al., 1997). Interestingly, paternal deletion of H19-DMR, although with H19 activation and decreased Igf2 expression, results in overall normal growth in the mouse (Leighton et al., 1995; Thorvaldsen et al., 1998). Taken together, the H19-Igf2 locus influences embryonic development probably through regulating the expression of $I g f 2$, which plays important roles in embryonic and placental development (Burns and Hassan, 2001; Morrione et al., 1997; Sibley et al., 2004).

The Dlk1-Dio3 is another essential imprinted region for embryonic development, in which, Gtl2 and $D l k 1$ are reciprocally expressed (Gtl2 is expressed from maternal allele, while $D l k 1$ is expressed exclusively from the paternal allele) (Schmidt et al., 2000; Takada et al., 2000) and controlled by intergenic germline-derived (IG)-DMR (Lin et al., 2003; Takada et al., 2002). Deletion of $I G$-DMR from maternal chromosome leads to loss of imprinting (LOI) in this locus and causes embryonic lethal after E16, but paternal deletion does not change the imprinting state of the region, leading to normal embryonic development in the resultant mouse (Lin et al., 2003).

With the identification of the imprinted genes, scientific efforts have been made to remove the paternal barriers that prevent the normal development of mouse bi-maternal embryos. 
Interestingly, PG embryos, which contained one set of chromosome from a neonate-derived nongrowing (ng) oocyte with imprinting free and the other from a fully grown (fg) oocyte, successfully developed to normal-sized fetus with a well-developed placenta on day 13.5 of gestation, 3 days longer than previously reported for parthenogenetic development (Kono et al., 1996). Furthermore, using the same system, when ng oocytes harboring a 3-kilobase (kb) deletion covering $H 19$ transcription unit, the reconstructed parthenotes could develop to day 17.5 of gestation (Kono et al., 2002). Moreover, when ng oocytes carrying a 13-kb deletion including both H19 and H19-DMR, a small ratio of the parthenotes could develop to term and grow to adulthood (Kono et al., 2004). Surprisingly, the birth rate of the reconstructed bi-maternal embryos was greatly improved when both $H 19$-DMR and $I G$-DMR were deleted in the ng oocytes, indicating that H19-Igf2 and Dlk1-Dio3 imprinted clusters are the main paternal barriers to normal development of bi-maternal fetus.

Recently, we and others generated mouse androgenetic haploid embryonic stem cells (AGhaESCs) that can be used as "artificial spermatids" to generate alive semi-cloned (SC) mice through intracytoplasmic injection (ICAHCI) into mature oocyte (Li et al., 2012; Yang et al., 2012). We further optimized the SC technology through generation AG-haESCs carrying both $H 19$-DMR and $I G$-DMR deletions that can efficiently support the development of SC embryos (Zhong et al., 2015). Interestingly, oocyte-originated haploid ESCs, when removed both H19DMR and $I G$-DMR, could also be used as sperm replacement to produce bi-maternal mice efficiently (Li et al., 2016; Zhong et al., 2016). Importantly, AG-haESCs mediated SC technology combined with CRISPR-Cas9 opens new avenues for genetic analyses in vivo (Jiang et al., 2018; Li et al., 2018; Wang and Li, 2019; Wei et al., 2017; Zhong et al., 2015). However, it is still not clear how the $H 19$-DMR and $I G$-DMR coordinately regulate SC embryo development.

In this study, we systemically analyzed the roles of paternal $H 19$-DMR and $I G$-DMR in SC embryonic development by characterizing the $\mathrm{SC}$ embryos at different stages during prenatal development and found that the H19-DMR and $I G$-DMR are dispensable for the development of preimplantation of SC embryos. Meanwhile, we showed H19-DMR is essential for the development of SC embryos before mid-gestation and $I G$-DMR is critical for late-gestation. Further, we demonstrated that $13-\mathrm{kb}$ deletion of paternal $H 19$ covering both H19-DMR and $H 19$ gene-body can improve the SC embryonic development before mid-gestation through rescue of placental defects caused by AG-haESCs. Finally, we established AG-haESCs carrying triple deletions (TKO), including H19, H19-DMR and $I G$-DMR, that can further improve the efficiency of producing viable, normal-sized, and fertile SC mice through ICAHCI. 


\section{RESULTS}

\section{Paternal H19-DMR and IG-DMR are not essential for the pre-implantation development of} SC embryos.

In our previous work, we have shown that double deletions of $H 19$-DMR and $I G$-DMR (DKO) in mouse AG-haESCs significantly improve the birth rate of SC mice (Zhong et al., 2015).

Meanwhile, we found that DKO does not change the transcriptional and methylation profiles of haploid ESCs, suggesting their potential functions during embryonic development. Therefore, in this study, we sought to further define how H19-DMR and $I G$-DMR regulate development of SC embryo. To this end, we chose wide-type (WT) AG-haESCs (AGH-OG3) and DKO-AG-haESCs (H19 $\triangle{ }^{\mathrm{DMR}}-I G^{\triangle \mathrm{DMR}}$-AGH-OG3) carrying an Oct4-eGFP transgene, which were derived in our previous studies (Yang et al., 2012; Zhong et al., 2015). PCR and eGFP fluorescence activation analyses confirmed that both cells carried expected transgene and deletions (Figures S1A and S1B). The haploidy of AG-haESCs was stably maintained by fluorescence-activated cell sorting (FACS)-based enrichment of haploid cells every 4-6 passages (Figure S1C). We first checked the expression of the imprinted genes regulated by H19-DMR and IG-DMR through quantitative reverse transcription PCR (RT-qPCR) analysis and found, as expected, H19 and Gtl2 were downregulated in DKO haploid cells, while Igf2 and Dio3 were up-regulated (Figure S1D). We then analyzed the transcriptomes of both cells and found that, consistent with previous results (Zhong et al., 2015), WT and DKO haploid cells exhibited a high correlation based on all expressed genes $(\mathrm{R}=0.99)$ (Figure $\mathrm{S} 1 \mathrm{E})$ and all known imprinted genes $(\mathrm{R}=0.99)$ (Figure $\mathrm{S} 1 \mathrm{~F})$. Interestingly, Fthl17, the X-linked maternal imprinted gene that may contribute to the early sex differentiation before gonadal differentiation (Kobayashi et al., 2010), was also downregulated in DKO cells (Figures S1D and S1F). Together, removal of H19-DMR and IG-DMR in AG-haESCs could mimic the methylation state of $H 19$-DMR and $I G$-DMR in sperm that lost during haploid ESC derivation and passaging in 2i medium (Choi et al., 2017; Yagi et al., 2017; Yang et al., 2012) (Figure S1G), leading to DKO haploid ESCs with decreased expression of $H 19$ and Gtl2, two critical paternal imprinted genes for embryonic development.

We next investigated whether H19-DMR and $I G$-DMR deletions are benefit to pre-implantation development of SC embryos generated by injection of WT or DKO AG-haESCs into mature oocytes (ICAHCI). The results showed that the efficiency of high-quality blastocysts was similar among sperm carrying Oct4-eGFP transgene (control), WT and DKO AG-haESCs (Figures 1A and 1B). To further characterize the similarity of blastocysts produced from different haploid cells, we separated inner cell mass (ICM) and trophectoderm (TE) and carried out low-cell-number (about 10) RNA-seq analysis (Figures S2A-S2C). Hierarchical clustering and principal component analysis (PCA) analyses of transcriptome data showed that all ICM samples were clustered together, but separated from TE samples which were also exclusively clustered together, indicating that the transcriptome differences between all samples are mainly caused by their different lineage origins (Figures 1C and S2D). Pearson correlation analysis further confirmed the similarity among different blastocysts (Figure 1D). Consistently, all imprinted genes exhibited similar expression patterns (Figure 1E). Moreover, the expression levels of H19, Igf2, Gtl2 and $D l k 1$ were extremely low in all tested ICM and TE samples (Figures $1 \mathrm{E}$ and $1 \mathrm{~F}$ ), suggesting that H19-Igf2 and Dlk1-Dio3 imprinted clusters may function during post-implantation development of SC embryos. Therefore, we examined the expression of H19, Igf2, Gtl2 and Dlk1 in control and 
SC embryos after implantation and found gradually increased expression of four genes following embryonic development from E3.5 to E9.5, implying that DKO could rescue transcriptional defects of $H 19$ and Igf2 in SC embryos from WT haploid cells (Figure 1G). These data demonstrate that DKO are not essential for pre-implantation development of SC embryos.

\section{$H 19$ and $I G$-DMR deletions (DKO) significantly improve the developmental potential of SC embryos during mid-gestation.}

We next examined the effect of DKO on prenatal development by transplantation of 2-cell SC embryos produced through ICAHCI of WT or DKO haploid cells into oviducts of pseudopregnant females (Figure 2A). A total of $2173 \mathrm{WT}$ and 1352 DKO SC two-cell embryos were transplanted. Dissection of SC embryos in pregnant females day-by-day from E6.5 to E12.5 revealed that DKO SC embryos implanted at significantly higher efficiency compared with WT SC embryos (41.9\% vs 34.6\%) (Figure 2B). Meanwhile, the developmental potential of DKO SC was significantly higher than WT SC embryos at E12.5 (Figure 2C). Moreover, higher frequency of abnormal embryos (degeneration or morphological abnormalities) displayed in WT group starting from E8.5 (Figures 2D and 2E). Interestingly, we found that most abnormal DKO SC embryos degenerated by E10.5 and almost all alive DKO SC embryos on E10.5 could develop to term in vivo (Figure 2C), whereas a high ratio of WT SC embryos showed both developmental retardation and degeneration and continued to display developmental failure at the following stages (Figures $2 \mathrm{~F}$ and $2 \mathrm{G}$ ), suggesting that DKO rescue the developmental defects of SC embryos before mid-gestation.

We next carefully compared the morphological differences between DKO and WT SC embryos. We observed that a high ratio of WT SC fetuses was alive with normal heartbeat on E8.5, but most of them exhibited developmental arrest starting from day 10.5 of gestation (Figure 3A).

Particularly, both fetal and placental weights were significantly lower in WT SC conceptus than those of the DKO SC conceptus on E12.5 (Figure 3B). Interestingly, the blood vessel of amniotic sac and umbilical cord were poorer and shorter in the WT SC conceptus, which may influence the nutrient and gas-exchange with mother (Figure 3C). The mature placenta contains three main layers: the labyrinth, which constitutes the main nutrient and gas-exchange surface; the basal zone, which consists of spongiotrophoblast, glycogen cells and different giant cell subtypes; and the maternally derived decidua (Rossant and Cross, 2001). Hematoxylin eosin (HE) staining analysis of placentas indicated that the WT SC placentas had smaller total areas and increased ratio of decidual areas compared with DKO ones (Figures 3D and 3E). Besides, we found that the glycogen cells in the basal zone was dramatically reduced in WT placenta (Figure 3D).

We next assessed the expression of critical imprinted genes in placentas via RT-qPCR. As expected, $H 19$ was overexpressed whereas $\operatorname{Ig} f 2$ expression was significantly decreased in WT placenta (Figure 3F), which may account for the reduced glycogen cells in WT SC placenta (Lopez et al., 1996). Igf2 is an important regulator in maintenance of the balance between supply and demand systems that is crucial to fine-tune mammalian growth (Angiolini et al., 2011; Constancia et al., 2005). We then tested the expression of glucose transporter genes (Slc2al and Slc2a3) and amino acid transporter genes (Slc38a1, Slc38a2, and Slc38a4) in E12.5 placentas. The results showed that Slc2a3 and Slc38a2 were significantly increased in the WT SC placenta (Figure S3A), probably leading to increased nutrient transfer that adapts to the nutrient supply to fetal demand (Constancia et al., 2005). Meanwhile, we also found that estrogen receptor (Esrl and 
$E s r 2)$ and androgen receptor $(A r)$ were highly expressed in WT placenta, whereas the inactivator of testosterone and estrogen (hydroxysteroid 17-beta dehydrogenase 2, Hsd17b2) had no difference (Figure S3A), consistent with previous observations that maternal androgen excess reduces placental and fetal weights (Sun et al., 2012). Besides, WT SC fetus died gradually from E11.5 to E15.5 (Figures S3B and S3C) and only two from a total of 308 WT SC (0.6\%) derived from AGH-OG3 cells with late passages (over passage 40) survived on E18.5 (Figures S3D and $\mathrm{S} 3 \mathrm{E})$. These results indicate that the developmental failure of WT SC embryos is mainly caused by placental defects, which can be rescued by $H 19$-DMR and $I G$-DMR deletions.

\section{IG-DMR deletion alone fails to rescue the developmental retardation of SC embryos before}

\section{E12.5.}

Interestingly, we observed that all dead embryos (E12.5) from WT AG-haESCs lost DNA methylation at the H19-DMR (Figures $3 \mathrm{G}$ and $3 \mathrm{H}$ ). In contrast, loss of DNA methylation at the $I G$-DMR appeared in both live and dead embryos on E12.5 (Figures $3 \mathrm{G}$ and $3 \mathrm{H}$ ). These results suggest that H19-DMR methylation is essential for SC embryonic development after implantation, while $I G$-DMR may not be very important before mid-gestation. To further explore their roles in SC embryonic development, we firstly deleted the $4 \mathrm{~kb}$ region of $I G$-DMR (Lin et al., 2003) in AGH-OG3 cells using CRISPR/Cas9 technology (Figure 4A). Two single guide RNAs (sgRNAs) targeting upstream and downstream of the region were designed and ligated to pX330-mCherry plasmid (Wu et al., 2013) and transfected into haploid cells, leading to two stable cell lines (termed $I G^{\triangle \mathrm{DMR}}$-AGH-OG3-1 and 2) (Figure S4A). Interestingly, these two cell lines were free of DNA methylation at H19-DMR (Figure S4B). ICAHCI analysis indicated that $I G$-DMR deletion did not improve developmental potential of SC embryos, and the percentage of abnormal SC embryos was comparable to that of WT SC embryos (Figure 4B). Meanwhile, the alive E12.5 fetus of $I G^{\triangle \mathrm{DMR}}$ exhibited growth-retarded phenotypes in both embryos and placentas (Figures 4C and 4D). Further, placental defects were more severe than WT fetus (Figures 4D-4F and S4C). Consistently, DNA methylation at $H 19$-DMR was absent in $I G^{\triangle \mathrm{DMR}} \mathrm{SC}$ embryos (Figures S4D and S4E), resulting in misexpression of $H 19$ and $I g f 2$ in $I G^{\triangle \mathrm{DMR}} \mathrm{SC}$ placentas (Figure $4 \mathrm{G}$ ). As expected, SC embryos with paternal $I G$-DMR deletion alone rarely developed to day 18.5 gestation (Figure $4 \mathrm{H}$ ). These results indicate that $I G$-DMR deletion in AG-haESCs is dispensable for the early stages of SC embryo development.

\section{$H 1{ }^{\Delta 13 \mathrm{~kb}}$ rescues developmental defects of $\mathrm{SC}$ embryos at mid-gestation.}

Having demonstrated that $I G$-DMR is not critical for SC embryonic development before midgestation, we next examined the role of H19-DMR in SC embryonic development. Due to that H19-DMR deletion cannot totally repress paternal $H 19$ expression and results in reduced Igf2 expression (Thorvaldsen et al., 1998; Thorvaldsen et al., 2002), to exclude the influence of paternal $H 19$ expression on the development of SC embryos, we attempted to remove the 13-kb region of $H 19$ that includes the gene body and the DMR in AG-haESCs (AGH-OG3) (Figures 5A

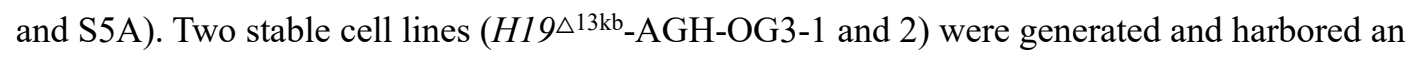
abnormal methylation status at $I G$-DMR similar to AGH-OG3 cells (Figures S5B and S5C). ICAHCI analysis showed that $H 19^{\triangle 13 \mathrm{~kb}}$ led to comparable developmental potential of SC to DKO AG-haESCs (Figure 5B) at E12.5, probably through rescue of placental defects (Figures 5C-5F, $\mathrm{S} 5 \mathrm{D}$ and S5E). Consistently, the expression of imprinted genes in $H 19^{\triangle 13 \mathrm{~kb}}$ placentas were similar 
to those in $H 19^{\triangle \mathrm{DMR}}-I G^{\triangle \mathrm{DMR}}$ DKO placentas (Figure 5G). Interestingly, we observed fewer abnormal embryos in $H_{19}{ }^{\triangle 13 \mathrm{~kb}}$ group compared to DKO group, probably due to slightly higher level of $I g f 2$ expression in $H 19 \triangle 13 \mathrm{~kb}$ embryos (Figure 5G).

We next checked the developmental potential of $H 1{ }^{-13 \mathrm{~kb}} \mathrm{SC}$ fetus at late-gestation. Compared to DKO SC embryos, the percentage of $H 19^{\triangle 13 \mathrm{~kb}} \mathrm{SC}$ embryos which normally developed to E18.5 was significantly reduced, accompanied with higher frequency of abnormal development (Figure $5 \mathrm{H}$ ). As expected, the survived $H 1{ }^{\triangle 13 \mathrm{~kb}} \mathrm{SC}$ embryos showed higher fetal and placental weights compared to DKO embryos (Figure 5I). Notably, we observed that the DNA methylation at $I G$ DMR lost in all abnormal $H 1{ }^{\Delta} \triangle^{13 \mathrm{~kb}}$ embryos on E18.5 (Figures 5J and 5K). Furthermore, growthretarded $H 19^{13 \mathrm{~kb}} \mathrm{SC}$ embryos with low methylation of $I G$-DMR (Figures S6A and S6B) exhibited enhanced Gtl 2 expression but repressed Dlk1 and Dio3 expression in different tissues (Figure S6C), indicating the critical roles of Dlk1-Dio3 imprinted cluster in late-gestation development.

\section{$H 19 \triangle^{13 \mathrm{~kb}}$ rescues developmental defects of $\mathrm{SC}$ embryos at mid-gestation through rescue of placental defects.}

Since different deletions resulted in different phenotypes in placentas at E12.5, we then sought to explore whether deletions changed the gene expression in the placenta by performing RNA sequencing (RNA-seq) analysis. To do this, we collected E12.5 placentas of five groups (three

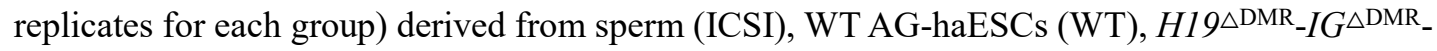

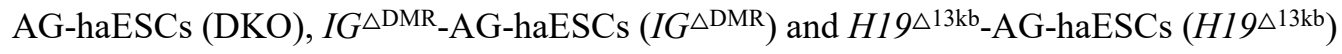
respectively. The results indicated that WT and $I G^{\triangle \mathrm{DMR}}$ placentas were clearly clustered together, and exhibited transcriptome patterns different from ICSI, $H 19^{\triangle 13 \mathrm{~kb}}$ and DKO placentas (Figures 6A, S7A and S7B). These results were consistent with the observations that $H 19^{\triangle 13 \mathrm{~kb}}$ and DKO significantly rescued the placental defects, while $I G^{\triangle \mathrm{DMR}}$ showed severe placenta defects as WT (Figures 4C-4F and 5C-5F). We further compared the expression patterns of imprinted genes and found that two groups of imprinted genes were differentially expressed between $\mathrm{DKO} / H 1{ }^{\Delta}{ }^{13 \mathrm{~kb}} / \mathrm{ICSI}$ and $I G^{\Delta \mathrm{DMR}} / \mathrm{WT}$, including genes involved in H19-Igf2 and Dlkl-Dio3 imprinted clusters (Figures 6B and 6C). Moreover, we also found three classes of genes which showed differential expression levels among the five groups of placentas (Figure 6D). Class I ( $n=$ 64) showed lower expression in $I G^{\triangle \mathrm{DMR}} /$ WT compared with $\mathrm{DKO} / H 19^{\triangle 13 \mathrm{~kb}} / \mathrm{ICSI}$ and was associated with cell cycle; Class II $(n=137)$ showed higher expression in $I G^{\triangle \mathrm{DMR}} / \mathrm{WT}$ and was enriched for developmental genes; Class III $(n=55)$ only expressed in WT and is associated with chromatin assembly (Figures 6D and 6E). Interestingly, we noticed that some Hox genes were abnormally activated in $I G^{\triangle \mathrm{DMR}} / \mathrm{WT}$ (Figure $6 \mathrm{~F}$ ), implying that they may be involved in abnormal $\mathrm{SC}$ embryonic development. A previous study has shown that $H 19$ and $\operatorname{Ig} 2$ maintain inactive during the first cell fate decision (ICM and TE formation), while they are specifically activated in visceral endoderm (VE) cells that mainly contribute to placental development, but not in epiblast (Epi) cells that develop into embryonic tissues (Figure 6G) (Zhang et al., 2018). These observations suggest that $H 19$ and H19-DMR may play a role in repression of developmentrelated genes in placenta. In summary, $I G^{\triangle \mathrm{DMR}}$ cannot rescue the abnormal gene expression in WT, including imprinted genes and other functional genes in E12.5 placentas, which may lead to severe developmental defects of SC embryos; in contrast, DKO and $H 19^{\Delta 13 \mathrm{~kb}}$ correct the misexpression of imprinted genes and other important functional genes in placenta, which contributes to the normal development in placenta. Taken together, these results provide strong 
evidence to demonstrate that H19-Igf2 locus on the paternal genome plays a key role in the SC embryonic development before mid-gestation, whereas Dlk1-Dio3 locus on the paternal genome is indispensable for normal embryonic development of late-gestation.

\section{Triple deletions of $H 19, H 19-D M R$ and $I G$-DMR further improve the developmental potential of SC embryos.}

Given that $H 1{ }^{\triangle} \triangle^{13 \mathrm{~kb}}$ can give rise to better development compared to DKO group on E12.5

(Figure 5B), we asked whether combined deletions between $H 19^{\triangle 13 \mathrm{~kb}}$ (including $H 19$ and H19DMR) and $I G$-DMR (TKO-AG-haESCs) could further improve full-term developmental potential of SC embryos. Two stable cell lines were generated through removal of $I G$-DMR in $H 19^{\Delta 13 \mathrm{~kb}}$

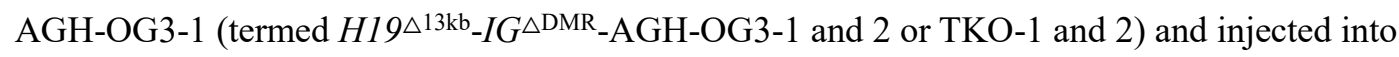
oocytes to produce mice (Figures 7A, 7B, S8A and S8B). The results showed that TKO-1 exhibited comparable developmental efficiency at E18.5 to DKO cells, while TKO-2 cells displayed a significantly higher efficiency (Figure 7B). Interestingly, TKO SC embryos exhibited remarkably higher body and placenta weights than DKO fetus, reaching to the levels of ICSI pups (Figures 7C and 7D). These could be caused by total deletion of paternal $H 19$, resulting in decreased $H 19$ expression in major organs during embryonic development (Figure 7E).

Importantly, the birth rate and body weight of TKO-2 newborn pups were notably higher than DKO (Figures 7F-7H). Furthermore, the growth profiling analysis showed that TKO-2 enhanced the growth of SC mice (Figure 7I). Consistently, removal of $H 19$ gene-body in another DKO cell line (termed $H 1{ }^{\triangle \mathrm{DMR}_{-}}-\mathrm{G}^{\triangle \mathrm{DMR}}$-AGH-2 or O48) generated previously (Zhong et al., 2015) resulted in a stable cell line termed O48-TKO, from which, SC pups with higher birth weight were produced (Figures S8C-S8E). Finally, we tested the reproductive ability of TKO SC mice by collecting mature oocytes from superovulated 4-weeks-old SC mice. The average number of oocytes from each mouse was similar among TKO, DKO and ICSI groups (Figures S8F and S8G). Besides, the concentration of luteinizing hormone ( $\mathrm{LH}$ ) in plasma of adult mice (4-month old) were also comparable between SC and control mice (Figure S8H). Moreover, TKO mice grew to adulthood and reproduced normally (Figure S8I). Genotyping analysis of a total of 9 live-born pups from 2 litters of progeny delivered by TKO SC mice showed that 3 carried the $13 \mathrm{~kb}$-deletion of $H 19$ (Figure S8J). All pups harboring maternal deletion of $I G$-DMR died shortly after birth, consistent with previous reports that maternal transmission of the $I G$-DMR deletion causes postnatal or neonatal lethality (Lin et al., 2003; Zhong et al., 2015). Taken together, these results demonstrate that TKO can further improve prenatal and postnatal development of SC embryos. 


\section{DISCUSSION}

Previous studies have shown that ng oocytes carrying both $H 19$ and $I G$-DMRs can efficiently support bi-maternal embryonic development after fusion with a mature oocyte (Kawahara et al., 2007). Meanwhile, we showed that removal of these two DMRs in AG and PG-haESCs can results in high-efficiency generation of SC and bi-maternal mice respectively through injection into oocytes (Zhong et al., 2016; Zhong et al., 2015). Moreover, we found that $H 19$ and $I G$-DMR deletions do not change the transcriptional and methylation profiles in haESCs (Zhong et al., 2016; Zhong et al., 2015), implying that $H 19$ and $I G$-DMRs may function during embryonic development. However, it is still largely unknown how $H 19$ and $I G$-DMRs coordinately regulate SC embryonic development. In this study, we show that $H 19$-DMR and $I G$-DMR temporally regulate SC embryo development (Figure 7J). H19-DMR and $I G$-DMR are dispensable for the preimplantation development of SC embryos. H19-DMR is indispensable for mid-gestation development, while $I G$-DMR plays a critical role in late-gestation development. H19-DMR is important for placental development before mid-gestation and double deletions of $H 19$ and H19DMR further improve SC embryonic development at mid-gestation, probably through regulation of Igf2 expression. Importantly, triple knockout of $H 19, H 19$-DMR and $I G$-DMR further improve the prenatal and postnatal development of SC mice.

Mice carrying paternal $H 19$-DMR or $I G$-DMR deletion that partially mimics methylation state of DMR (Barlow and Bartolomei, 2014) have been generated a decade ago (Lin et al., 2003; Thorvaldsen et al., 2002), displaying minimal if any adverse phenotypes. Therefore, it is expectable that sperm with both $H 19$-DMR and $I G$-DMR deletions obtained through multiple rounds of breeding between two mutant mouse lines may also result in overall normal embryonic development, thus impeding the study of coordinated regulation between two imprinted loci in embryonic development. In contrast, AG-haESCs with low methylation level especially in imprinted regions results in abnormal embryonic development upon injection into oocytes (Yang et al., 2012). Interestingly, after removal of H19-DMR and IG-DMR in AG-haESCs to rescue the methylation lost at DMRs, the resultant DKO-AG-haESCs can efficiently support normal SC embryonic development, leading to alive pups with a high birth rate comparable to that of round spermatid injection (ROSI) (Zhong et al., 2015). Taken together, these results suggest that AGhaESCs, acting as the sperm replacement, can be used to study the imprinting function in vivo. Using this technology, our current study reveals the temporal regulation of paternal H19-Igf 2 and Dlk1-Dio3 during embryonic development, providing a strong evidence to demonstrate AGhaESCs as an unique tool to study genomic imprinting in vivo ( $\mathrm{Li}$ and $\mathrm{Li}, 2019$ ).

Due to the accessibility of genomic modifications in AG-haESCs, this technology may be extended to different topics of imprinting studies in vivo, such as: 1) identification of maternal imprinted genes that are critical for embryonic development, whose deletion in AG-haESCs may result in SC embryonic lethal; 2) investigation of control elements of the imprinted locus through one-step generation of SC mice carrying different modifications in the imprinted locus by respective injection of AG-haESCs with corresponding mutations into oocytes; 3) characterization of the expression pattern of a imprinted gene during embryonic development through insertion of a standard tag endogenously in AG-haESCs; and 4) interaction analysis between different imprinted genes/loci. 
TKO-AG-haESCs generated in this study exhibit higher developmental potential and better postnatal development of SC embryos; these cells, combing with CRISPR-Cas9 technology, may further enhance genetic analyses in vivo (Li et al., 2018; Wang and Li, 2019; Wei et al., 2017). Further investigation into H19-Igf2, Dlk1-Dio3 or other loci will not only reveal mechanisms involved in embryonic development, but may also help to fine-tune the embryonic development for more efficient generation of SC mice.

\section{MATERIALS AND METHODS}

\section{Animals}

All mice were housed in individual ventilated cages (IVC) under specific-pathogen-free and a 12:12 h light/dark cycle condition. MII oocytes are all from B6D2F1 (C57BL/6ㅇ $\times$ DBA/2ð) female mice. The Oct4-eGFP males (C57BL/6 background) provided sperm for ICSI experiments. All the pseudopregnant foster mothers were ICR females. All animal procedures were performed under the ethical guidelines of the Shanghai Institute of Biochemistry and Cell Biology, Chinese Academy of Sciences, Shanghai, China.

\section{Plasmids construction}

To generate CRISPR-Cas9 plasmid for gene manipulation, specific sgRNAs were synthesized (Sunya, Shanghai), annealed, and fused to Bpil (Thermo Scientific ${ }^{\mathrm{TM}}$ ) linearized $\mathrm{pX} 330-m$ Cherry plasmid (Addgene\#98750). All inserted sgRNA sequences were validated by Sanger sequencing.

\section{Cell culture and transfection}

Mouse androgenetic haploid embryonic stem cells (AG-haESCs) of AGH-OG3 and H19 $\triangle \mathrm{DMR}_{-}$ $I G^{\triangle \mathrm{DMR}}$-AGH-OG3 were from our previous works(Yang et al., 2012; Zhong et al., 2015), respectively. These cells were cultured in DMEM (Merk) with 15\% FBS (Thermo Scientific ${ }^{\mathrm{TM}}$ ), Penicillin-Streptomycin, Non Essential Amino Acids, Nucleosides, L-Glutamine Solution, 2Mercaptoethanol, 1000 U/ml Lif, $1 \mu$ M PD03259010 (Selleck) and $3 \mu$ M CHIR99021 (Selleck). Cells were transfected using Lipofectamine 3000 reagent (Thermo Scientific ${ }^{\mathrm{TM}}$ ) according to the manufacturer's protocols.

\section{CRISPR/Cas9 mediated imprinted region deletion}

For constructing gene-edited AG-haESCs, cells were transfected with corresponding pX330$m$ Cherry plasmids, including specific sgRNAs (Table S1). 24 hours after transfection, the haploid cells expressing red fluorescence protein were enriched with flow cytometry (FACS AriaII, BD Biosciences) and plated at low density (about 8000 cells per well of 6-well plate). One week after plating, single colony was picked for derivation of gene manipulation AG-haESCs identified by PCR and Sanger sequencing. For the enrichment of haploid cells, cells were trypsinized, incubated with $15 \mu \mathrm{g} / \mathrm{ml}$ Hoechst 33342 (Thermo Scientific ${ }^{\mathrm{TM}}$ ) in $37^{\circ} \mathrm{C}$ water bath for 5-10 min, transferred through a $40-\mu \mathrm{m}$ cell strainer and enriched by sorting $1 \mathrm{~N}$ peak on AriaII.

\section{Genomic DNA extraction and genotyping}

Mouse tails and AG-haESCs were lysed by Mouse Direct PCR Kit (Bimake) according to the 
manufacturer's guidance. After centrifuge, 12,000 rpm for $10 \mathrm{~min}, 1-2 \mu \mathrm{l}$ supernatant of digested solution can be used as the PCR template. The genome extraction from mouse embryos or pups were using the TIANamp Genomic DNA Kit (TIANGEN), which used for genotyping or bisulphite sequencing.

\section{Bisulphite sequencing}

The bisulfite conversion was performed into one-step using the EZ DNA Methylation-GoldTM Kit (ZYMO research) for 500 ng genomic DNA, following the manufacturer's instructions. The recovery DNA products were amplified by a first round of nested PCR, following a second round using loci specific PCR primers (Table S1). The amplified products were purified by gel electrophoresis using Universal DNA Purification Kit (TIANGEN) and cloned into a pMD19-T vector (Takara). For each sample, more than fifteen $E$. coli clones were picked up for sequencing. The results were analyzed by DNA methylation analysis platform (http://services.ibc.unistuttgart.de/BDPC/BISMA/).

\section{RNA extraction and quantitative real-time PCR}

The total RNA of high quality was isolated from cells and tissues of embryos (E8.5, E9.5, E12.5 and E18.5) using TRIzol ${ }^{\mathrm{TM}}$ Reagent (Thermo Scientific ${ }^{\mathrm{TM}}$ ). To synthesize high-quality cDNAs for real-time PCR, a mount of 500 ng of total RNA was directly used as template with ReverTra Ace ${ }^{\circ}$ qPCR RT Master Mix (TOYOBO). To obtain high-quality RNA from embryos with low-cell number (E3.5, E6.5 and E7.5), samples were lysed in $150 \mu \mathrm{l}$ of $4 \mathrm{M}$ guanidine isothiocyanate solution (Thermo Scientific ${ }^{\mathrm{TM}}$ ) at $42{ }^{\circ} \mathrm{C}$ for $10 \mathrm{~min}$. Total RNA pellets were concentrated by UltraPure $^{\mathrm{TM}}$ Glycogen (Thermo Scientific ${ }^{\mathrm{TM}}$ ). To get enough amount of template for real-time PCR, two rounds of amplification were performed according to a reported protocol (Chen et al., 2017). Quantitative real-time PCR was performed on Bio-Rad CFX96 using the $S Y B R^{\circledR} q P C R$ Mix (TOYOBO) in triplicate. All gene expression was calculated based on the $2^{-\Delta \Delta \mathrm{Ct}}$ method after normalization to the transcript level of the internal standard gene, Gapdh. All the primer sequences are listed in (Table S1).

\section{RNA sequencing and analyzing}

To separate ICM and TE from E3.5 embryos(Liu et al., 2016), blastocysts were broken using micromanipulator and washed three times in HCZB. The zona pellucidae of blastocysts were digested with $0.5 \%$ pronase $\mathrm{E}$ (Sigma) for $10-15 \mathrm{~min}$ and washed three times again in HCZB. The embryos were then incubated in $\mathrm{Ca}^{2+}$-free $\mathrm{CZB}$ for $30 \mathrm{~min}$ in $37^{\circ} \mathrm{C}$, and the ICM and TE were separated into single cells by gently pipetting using a pipette with a diameter of $40-60 \mu \mathrm{m}$. Each sample was obtained about 10 cells from one embryo for amplification according the cell shape. ICM cells are small and out-of-shape, while TE cells are big and smooth. The cDNAs of low-input cells were harvested according previously protocols (Picelli et al., 2014). The cDNA libraries were prepared by SPAPK DNA Sample Pre Kit (Enzymatics) for sequencing using an Illumina HiSeq X Ten sequenator. Total RNA was isolated from mouse placentas using Dynabeads ${ }^{\mathrm{TM}}$ mRNA DIRECT $^{\text {TM }}$ Purification Kit (Thermo Scientific ${ }^{\mathrm{TM}}$ ). RNA-seq libraries were prepared by VAHTS mRNA-seq V3 Library Prep Kit for Illumina ${ }^{\circledR}$ (Vazyme), and then applied for deep sequencing on Illumina NovaSeq6000 platform. All RNA-seq reads were mapped to mm9 with TopHat (version 2.2.1). The mapped reads were further analyzed by Cufflinks and the expression levels for each 
transcript were quantified as Fragments Per Kilobase of transcript per Million mapped reads (FPKM).

\section{Intracytoplasmic AG-haESCs and sperms injection}

To generate SC embryos, AG-haESCs were treated with $0.05 \mu \mathrm{g} / \mathrm{ml}$ Demecolcine solution (Sigma) for 10-12 $\mathrm{h}$ and synchronized to $\mathrm{M}$ phase. These cells were trypsinized and suspended in HCZB medium. MII oocytes were collected from oviducts of superovulated B6D2F1 females (8-weekold). AG-haESCs were injected into the cytoplasm of MII oocytes in a droplet of HCZB medium containing $5 \mu \mathrm{g} / \mathrm{ml}$ cytochalasin B (Sigma) using a Piezo-drill micromanipulator. The reconstructed oocytes were cultured in CZB medium for $30 \mathrm{~min}$ and then activated for 5-6 $\mathrm{h}$ in $\mathrm{Ca}^{2+}$ free CZB with $\mathrm{SrCl}_{2}$. For ICSI experiments, the procedures were the same as described above, except that AG-haESCs were replaced by sperm. Following activation, all of the reconstructed embryos were cultured for $24 \mathrm{~h}$ in AA-KSOM (Merk) medium at $37{ }^{\circ} \mathrm{C}$ under $5 \%$ $\mathrm{CO}_{2}$ in air.

\section{Embryo transfer and cesarean section}

Reconstructed embryos were cultured in AA-KSOM medium until the two-cell or blastocyst stage. Thereafter, 14-16 (for ICSI) and 18-20 (for ICAHCI) two-cell embryos were transferred into each oviduct of pseudopregnant ICR females at 0.5 days post copulation. The post-implantation embryonic development (from E6.5 to E18.5) was assessed by an autopsy, and the embryos were quickly removed from the uteri and photographed immediately. Some E12.5 and E18.5 embryos and placentas were weighed after being dried with absorbent paper.

\section{Placenta histology analysis}

The placentas of E12.5 were fixed in $4 \%$ PFA overnight at room temperature, dehydrated with gradient concentration of alcohol, and embedded in paraffin and sectioned. Serial sections $(4 \mu \mathrm{m})$ prepared in the cross planes were mounted on slides and dewaxed in xylene and rehydrated in different concentration of alcohol, followed by staining with hematoxylin and eosin. In each placenta, five sections of different slides were picked up to calculate the total placenta area, decidual zone area, labyrinthine zone area, and basal zone area. The values were measured with ImageJ software, and the area is the average of five different sections of each sample.

\section{Collection of mature oocytes and measure of luteinizing hormone (LH)}

Control (ICSI), DKO and TKO female mice (4-week old) were superovulated with 4 international units of pregnant mare's serum gonadotropin (PMSG) for $48 \mathrm{~h}$ and then human chorionic gonadotropin (hCG) for $12 \mathrm{~h}$. Mature oocytes were collected from ampulla of the fallopian tube after superovulation. Plasma was collected from 4-month female mice using heparin as an anticoagulant. All samples were centrifuged for $15 \mathrm{~min}$ at $1000 \mathrm{~g}$ at $4{ }^{\circ} \mathrm{C}$ within $30 \mathrm{~min}$ and the supernatant was collected for the next assay. LH signals were detected from plasma using Mouse LH / Luteinizing Hormone ELISA Kit (LSBio), following the manufacturer's instructions.

\section{Statistical analysis}

For all figures, results shown are the average mean \pm standard error of the mean (s.e.m.).

Statistical testing was performed using GraphPad Prism 7. Significance was determined by two- 
tailed, unpaired Student's t-test. ${ }^{*} P<0.05,{ }^{* *} P<0.01, * * * P<0.001$, n.s means no significant difference.

\section{Data availability}

The RNA sequencing data of blastocysts and placentas from this study have been deposited in the Gene Expression Omnibus (GEO) under accession code GSE132254. The RNA sequencing data of AGH-OG3 and $H 19 \triangle \triangle^{\mathrm{DMR}}-I G^{\triangle \mathrm{DMR}}$-AGH-OG3 obtained from our previous work under the accession number GSE60072. The RNA sequencing data of ICM and TE of E3.5 and Epi and VE of E6.5 from previous work under the accession number GSE76505. All other data supporting the findings of this study are available from the corresponding author on reasonable request.

Compliance and ethics The authors declare that they have no conflict of interest.

Acknowledgements This study was partly supported by Genome Tagging Project, Fountain-Valley Life Sciences Fund of University of Chinese Academy of Sciences Education Foundation and grants from the Chinese Academy of Sciences (XDB19010204, OYZDJ-SSWSMC023 and Facility-based Open Research Program), the National Natural Science Foundation of China (31530048, 81672117, 31730062, 31821004 and 31601163), and Shanghai Municipal Commission for Science and Technology (16JC1420500, 17JC1420102, 17 JC1400900 and 17411954900).

Author contributions J.L. conceived of the project. Q.L. and Q.Y. performed the ICAHCI experiments. Y.L. and K.W. analyzed RNA-seq data. Q.L. and S.H. performed cell culture, histomorphology and molecular biological experiments. L.Z., W.L. and B.C. helped with embryo transplantation and the mouse experiments. Q.L., Y.L. and J.L. designed the experiments, analyzed the data and wrote the paper. All authors contributed to the manuscript. 


\section{FIGURES}

A

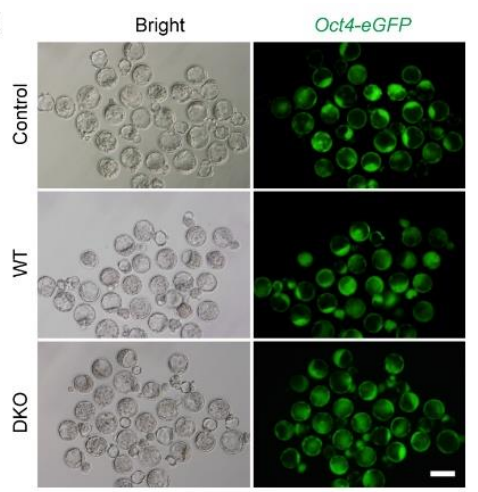

B

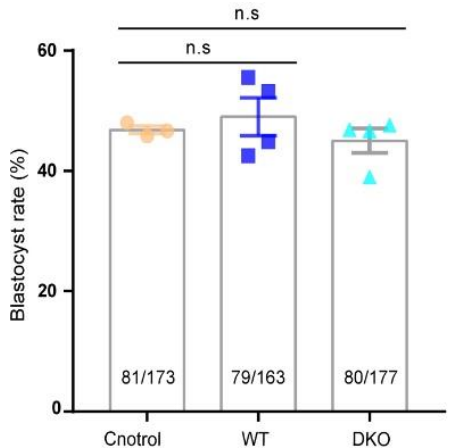

C

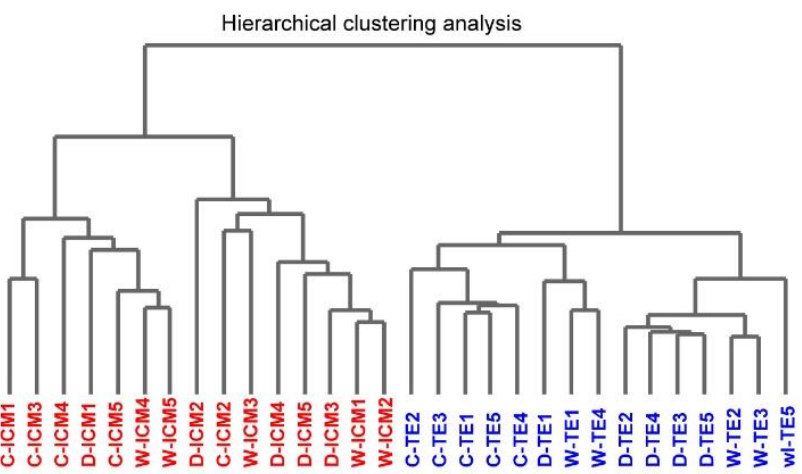

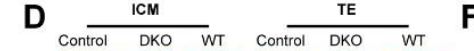

$\left.F^{80}\right]^{-10 \text { comol }} \quad$ ICM

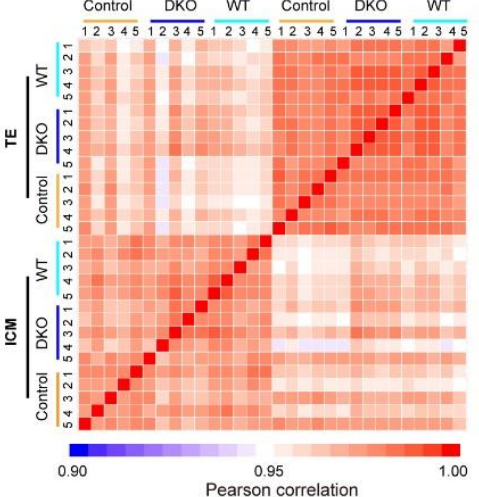

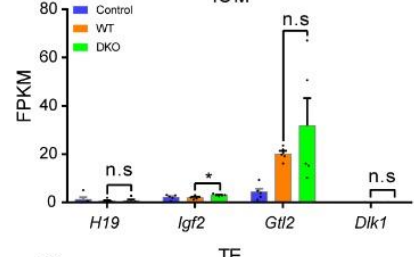

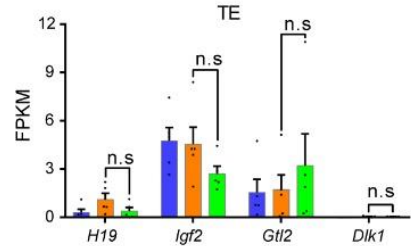

E
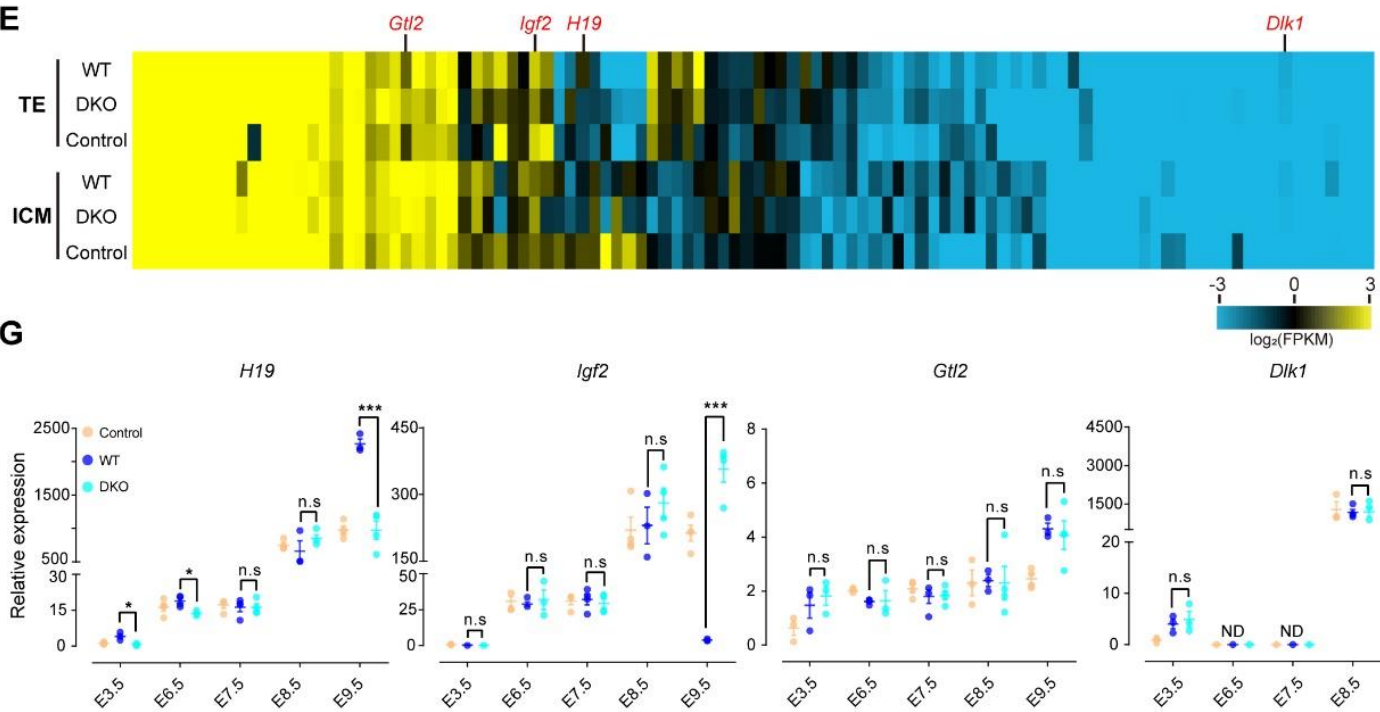

Gt/2

DIk1

Figure 1. Paternal $H 19$-DMR and $I G$-DMR are dispensable for the preimplantation development of SC embryos.

(A and B) Representative images of blastocysts (A) and the efficiency of blastocysts derived from sperm (control), WT and DKO-AG-haESCs (B), showing the similar developmental potential among different haploid cells. Numbers in the bars showing blastocysts/two-cells ratio. (C and D) The hierarchical clustering analysis (C) and correlation heatmap (D) of RNA-seq data from ICMs (5 replicates) and TEs (5 replicates) obtained through different haploid donors. C, control embryos derived from intracytoplasmic sperm injection (ICSI); W, embryos from intracytoplasmic WT-AG-haESC injection; D, embryos from DKO-AG-haESC injection. 
(E) The heatmap showing expression patterns of imprinted genes in TE (top) and ICM (bottom).

The gene expression levels were measured as $\log _{2}$ (FPKM).

(F) The expression levels of H19, Igf2, Gtl2 and Dlk1 in ICM and TE.

(G) Transcriptional analysis of $H 19, \operatorname{Ig} f 2, G t l 2$ and $D l k 1$ genes among control (ICSI), WT and DKO embryos from E3.5 to E9.5 ( $n=3$ embryos). The expression values were normalized to that of Gapdh. All error bars in B, F and G indicate the average mean \pm s.e.m. ${ }^{*} P<0.05,{ }^{* * *} P<$ 0.001. n.s, no significant difference.

A

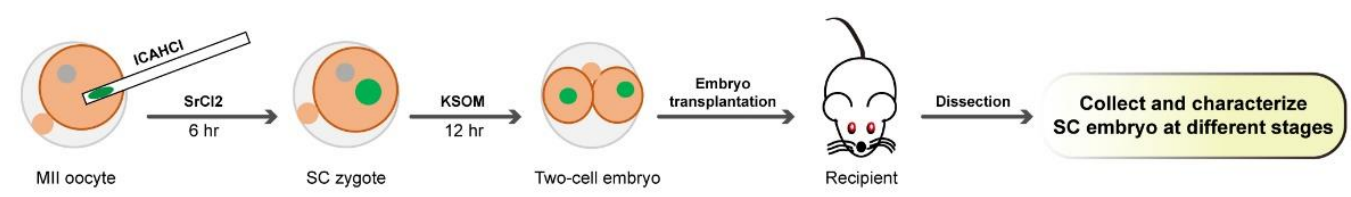

B

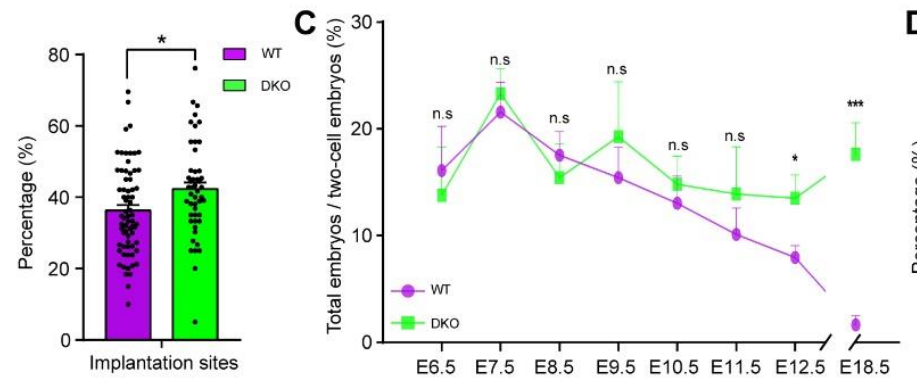

D

E
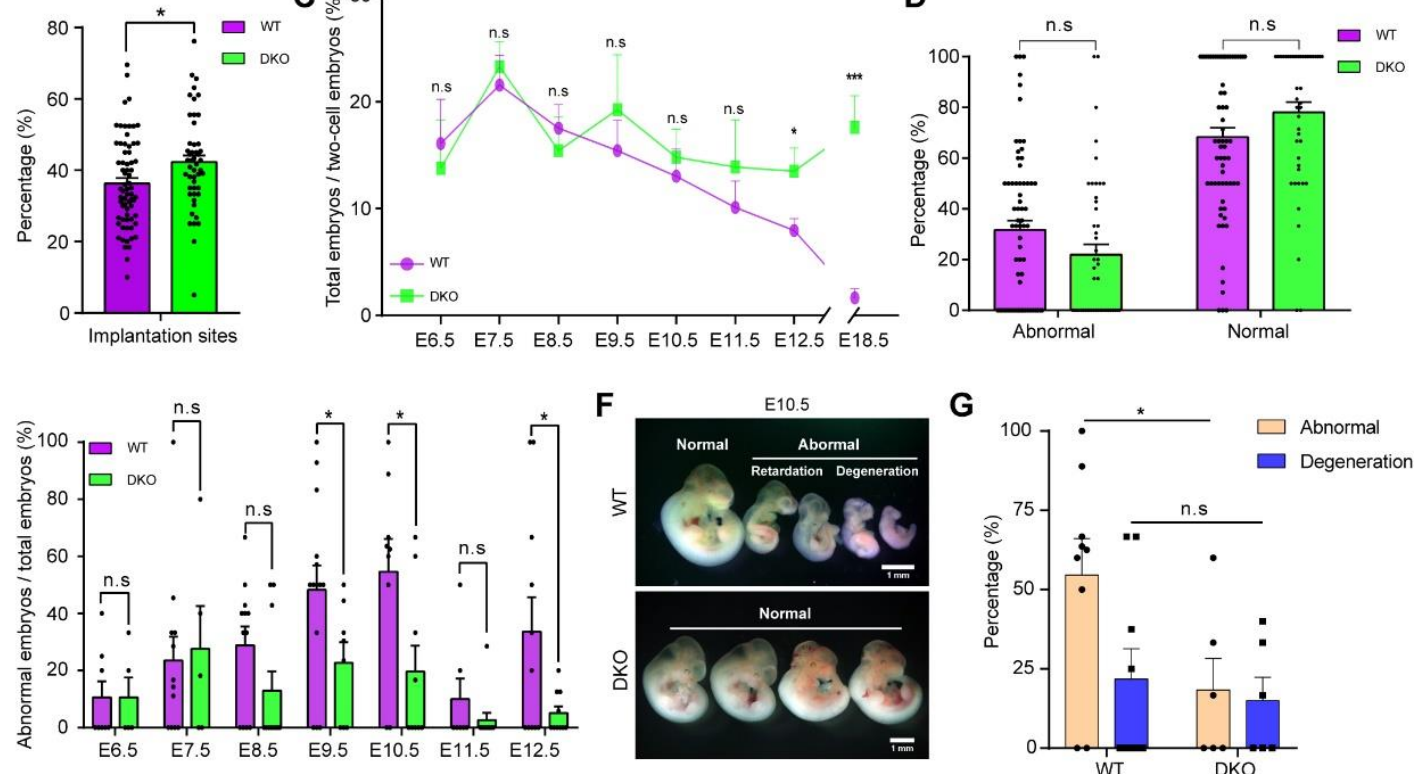

Figure 2. Developmental potential analysis of SC embryos at different stages.

(A) Schematic diagram of developmental potential analysis of SC embryos generated via ICHACI of WT or DKO AG-haESCs.

(B) Summary of the average implantation rate of WT ( $n=68$ recipients) and DKO ( $n=49$ recipients) SC embryos dissected from E6.5 to E12.5.

(C) Comparison of the individual developmental efficiency on different embryonic days between WT and DKO SC embryo.

(D) Summary of the total ratios of abnormal and normal SC embryo (from E6.5 to E12.5) derived from WT ( $n=68$ recipients) and DKO ( $n=49$ recipients).

(E) Comparison of the individual ratios of abnormal $\mathrm{SC}$ embryos on different embryonic days (from E6.5 to E12.5) between WT and DKO groups.

(F and G) Representative images of E10.5 SC embryos produced from WT (top panel) and DKO (bottom panel) AG-haESCs (F). The ratios of abnormal and degeneration embryos show in (G). The dark spots in B, D, E and G show one data from one recipient. All error bars in B-E and G indicate the average mean \pm s.e.m. ${ }^{*} P<0.05,{ }^{* * *} P<0.001$. n.s means no significant difference. 
A

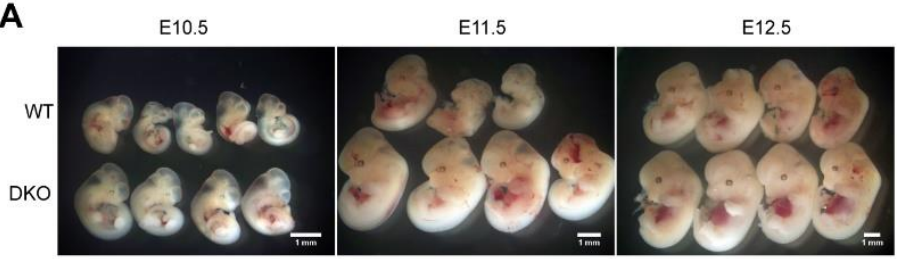

C

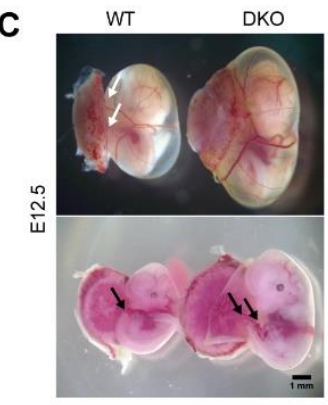

D

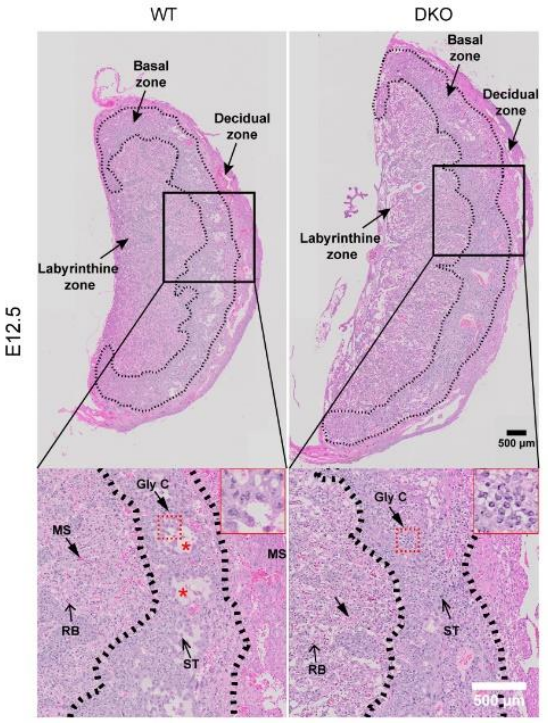

E

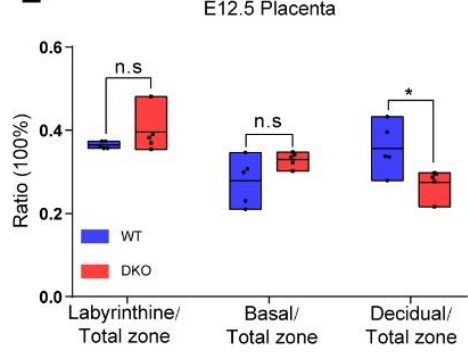

B

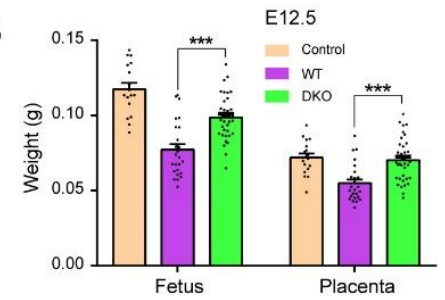

$\mathbf{F}$

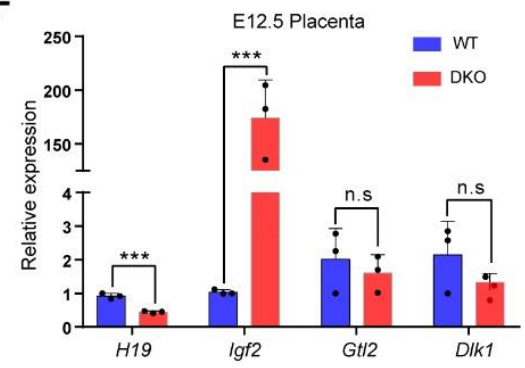

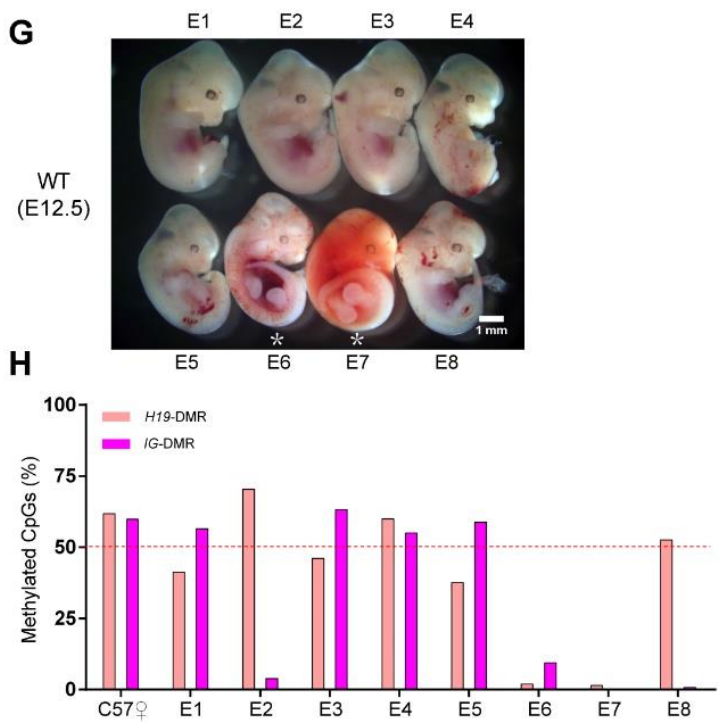

Figure 3. H19-DMR and $I G$-DMR double knock-out significantly rescue the retardation of SC embryos at mid-gestation.

(A) Representative images of alive SC embryos (E10.5 to E12.5) derived from WT (top panel) and DKO (bottom panel) AG-haESCs show retarded growth in WT group.

(B) The fetal and placental weights of E12.5 embryos derived from sperm (control, $n=17)$, WT ( $n$ $=25)$ and DKO $(n=36)$ AG-haESCs, showing severe retardation in WT embryos compared with DKO embryos.

(C) E12.5 conceptus derived from WT and DKO AG-haESCs. An obvious gap (white arrow) between the placenta and visceral yolk sac shows in WT fetus (top panel). The well-developed umbilical cord (black arrows) shows in the DKO fetus (bottom panel). Experiments were repeated five times independently, with similar results.

(D) Whole mount images of SC placentas (E12.5) (top panel). Black boxes in the top panel are magnified in bottom panel. The total placental area is smaller in the WT compared with DKO. Fewer glycogen cells (Gly C) exist in the basal zone of the placenta from the WT compared with DKO, shown in red dashed boxes. Red asterisk showed vacuolation in WT placenta. MS, maternal sinusoid; RB, red blood cells; ST, spongiotrophoblast. Experiments were repeated five times independently, with similar results. 
(E) The ratios of labyrinthine zone, basal zone and decidual zone to total placental areas of WT and DKO placentas (E12.5), indicating increased decidual area in WT placentas. 5 placentas for each group and at least 5 sections per placenta were analyzed. Middle lines are shown as the mean.

(F) Transcriptional analysis of H19, Igf2, Gtl2 and Dlk1 genes in WT and DKO placentas (3 placentas for each group). The expression values were normalized to that of Gapdh. Data are shown as the average mean \pm s.e.m. in $\mathrm{B}$ and $\mathrm{F} . * P<0.05, * * * P<0.001$. n.s means no significant difference.

( $\mathrm{G}$ and $\mathrm{H}$ ) Images of eight WT SC embryos $(\mathrm{G})$ and their methylation state of the H19-DMR and $I G$-DMR $(\mathrm{H})$. Embryos with a white asterisk are dead. The dotted line shows theoretical value of methylation, $50 \%$.

A

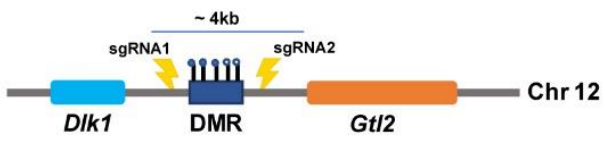

B

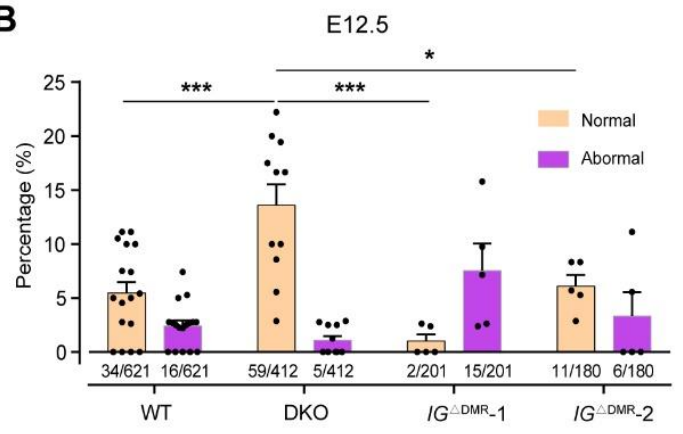

E

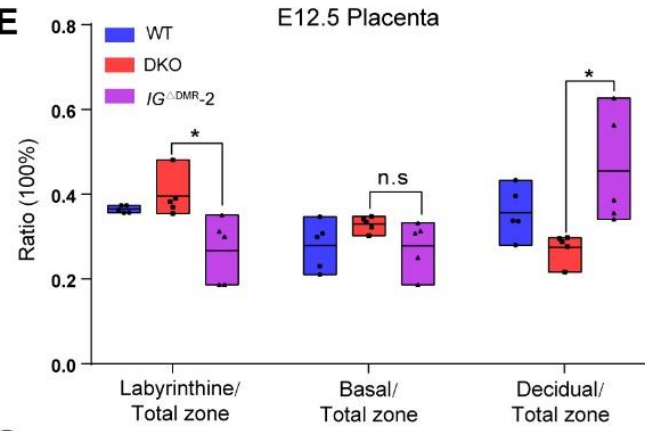

G

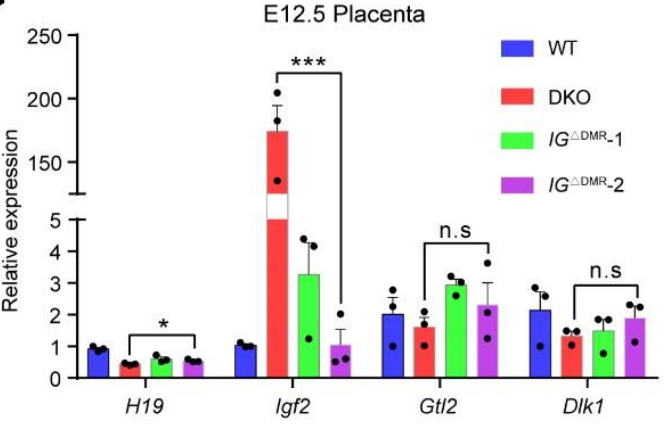

C

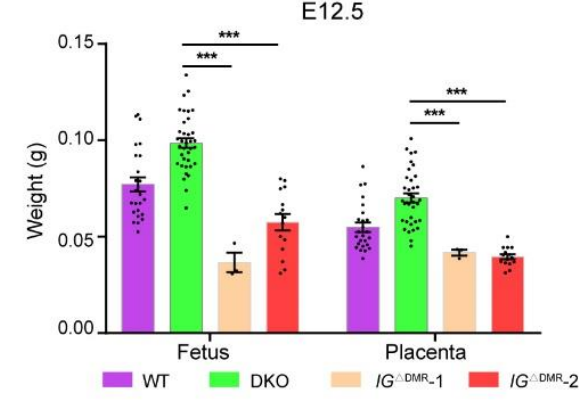

IG ${ }^{\triangle M R_{-}}-1$

IG ${ }^{\triangle M M R}-2$

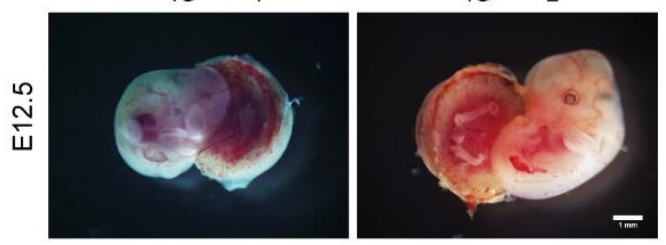

$\mathbf{F}$

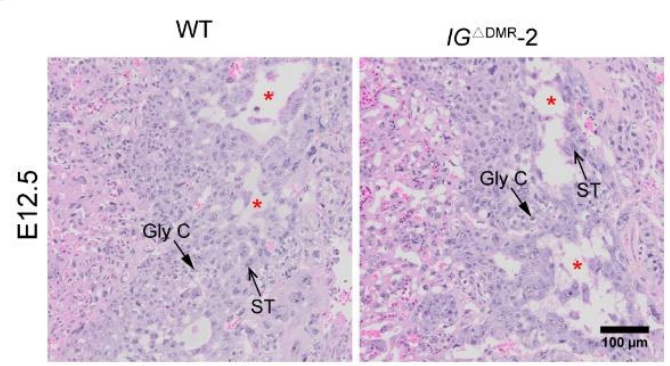

H

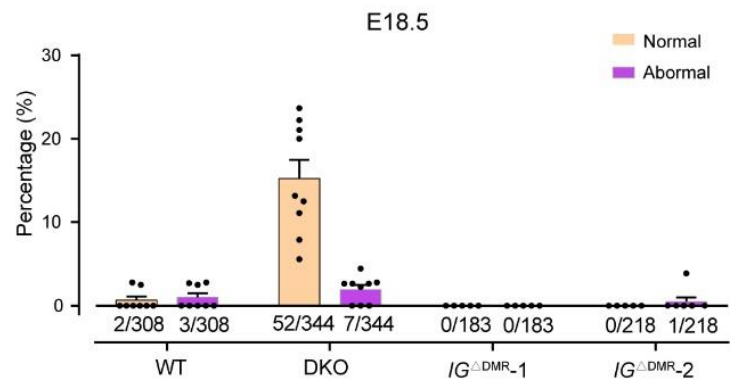

Figure 4. Deletion of $I G$-DMR alone fails to rescue the developmental retardation of SC embryos before E12.5. 
(A) Schematic diagram of sgRNAs targeting for the removal of $I G$-DMR. A dark blue bar represents the deleted region (about 4-kb).

(B) The ratios of normal and abnormal SC embryos (E12.5) in transferred 2-cell embryos derived from WT ( $n=17$ recipients), DKO ( $n=11$ recipients) and $I G^{\triangle \mathrm{DMR}}(n=5$ recipients for each cell line) AG-haESCs. Numbers under the bars indicate E12.5 embryos/transferred two-cell embryos. (C) The fetal and placental weights of E12.5 embryos derived from WT $(n=25)$, DKO $(n=36)$ and $I G^{\triangle \mathrm{DMR}}\left(n=5\right.$ for $I G^{\triangle \mathrm{DMR}_{-}} 1$ and $n=15$ for $I G^{\triangle \mathrm{DMR}_{-}}$) AG-haESCs, showing severe retardation in $I G^{\triangle \mathrm{DMR}} \mathrm{SC}$ embryos compared with $\mathrm{DKO}$.

(D) Severe developmental retardation shown in $I G^{\triangle \mathrm{DMR}} \mathrm{SC}$ fetuses (E12.5). Experiments were repeated five times independently, with similar results.

(E) The ratios of labyrinthine zone, basal zone and decidual zone to total placenta areas of WT, DKO and $I G^{\triangle \mathrm{DMR}}$ placentas (E12.5), indicating decreased labyrinthine zone and increased decidual area in $I G^{\triangle \mathrm{DMR}}$ placentas compared with DKO, $n=5$ placentas for each group and at least 5 sections per placenta analyzed. Middle lines are shown as the mean.

(F) Histological sections of the placentas from WT and $I G^{\triangle \mathrm{DMR}} \mathrm{SC}$ fetus on E12.5. Red asterisks show vacuolation in WT and $I G^{\triangle \mathrm{DMR}}$ placentas. Gly C, glycogen cells; ST, spongiotrophoblast. Experiments were repeated five times independently, with similar results.

(G) Transcriptional analysis of $H 19$, Igf2, Gtl2 and Dlkl genes in WT, DKO and $I G^{\triangle \mathrm{DMR}}$ placentas ( $n=3$ placentas for each group). The expression values were normalized to that of Gapdh.

$(\mathrm{H})$ The ratios of normal and abnormal SC embryos (E18.5) in transferred 2-cell embryos derived from WT ( $n=8$ recipients), DKO ( $n=9$ recipients) and $I G^{\triangle \mathrm{DMR}}(n=5$ recipients for each cell lines) AG-haESCs. Numbers under the bars indicate E18.5 embryos/transferred two-cell embryos. Data are shown as the average mean \pm s.e.m in B, C, G and H. $* P<0.05$, ${ }^{* * *} P<0.001$. n.s means no significant difference. 
A

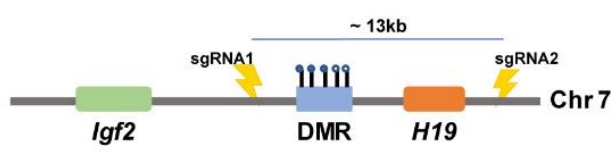

B

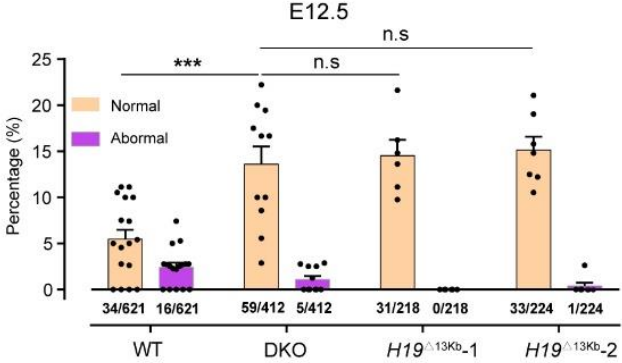

D

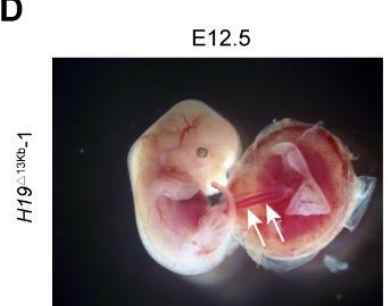

E
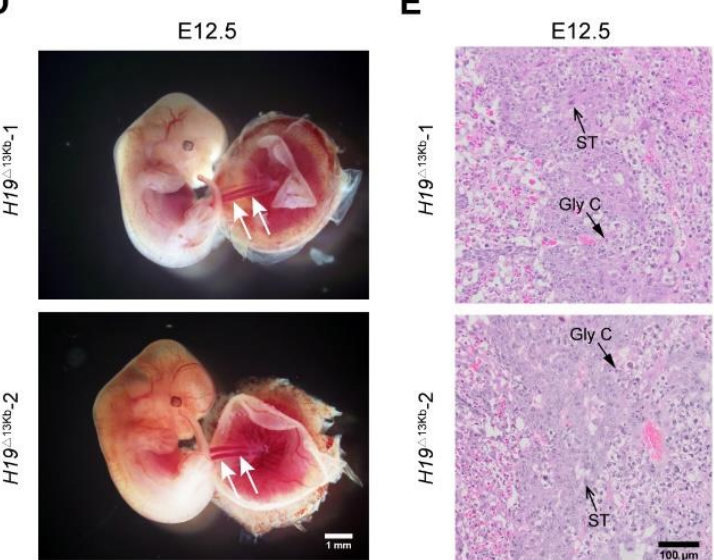

H

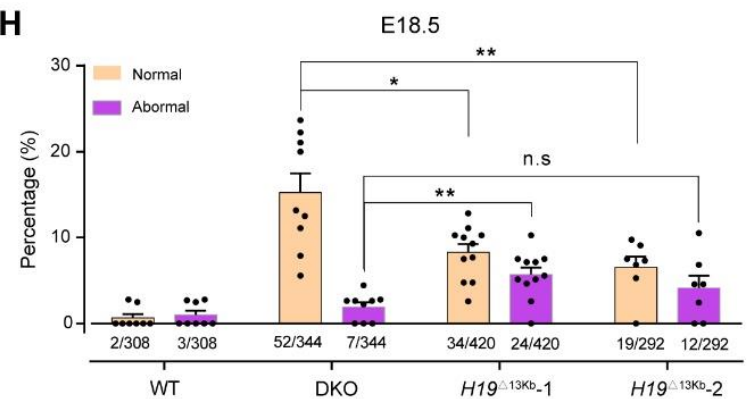

$\mathbf{J}$

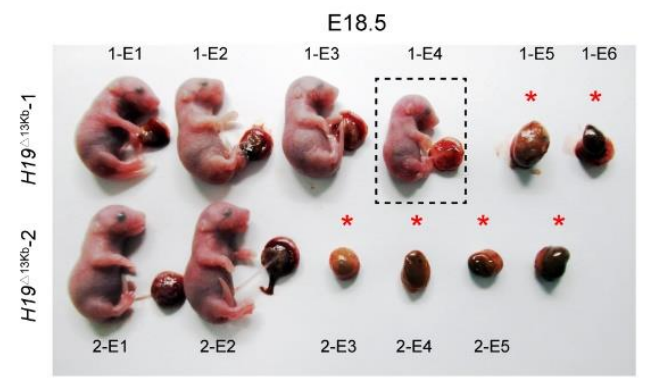

C

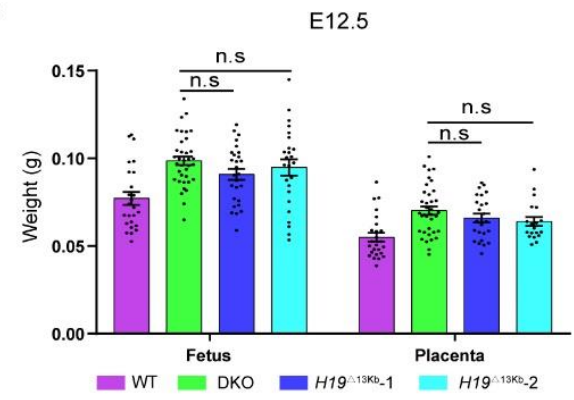

$\mathbf{F}$
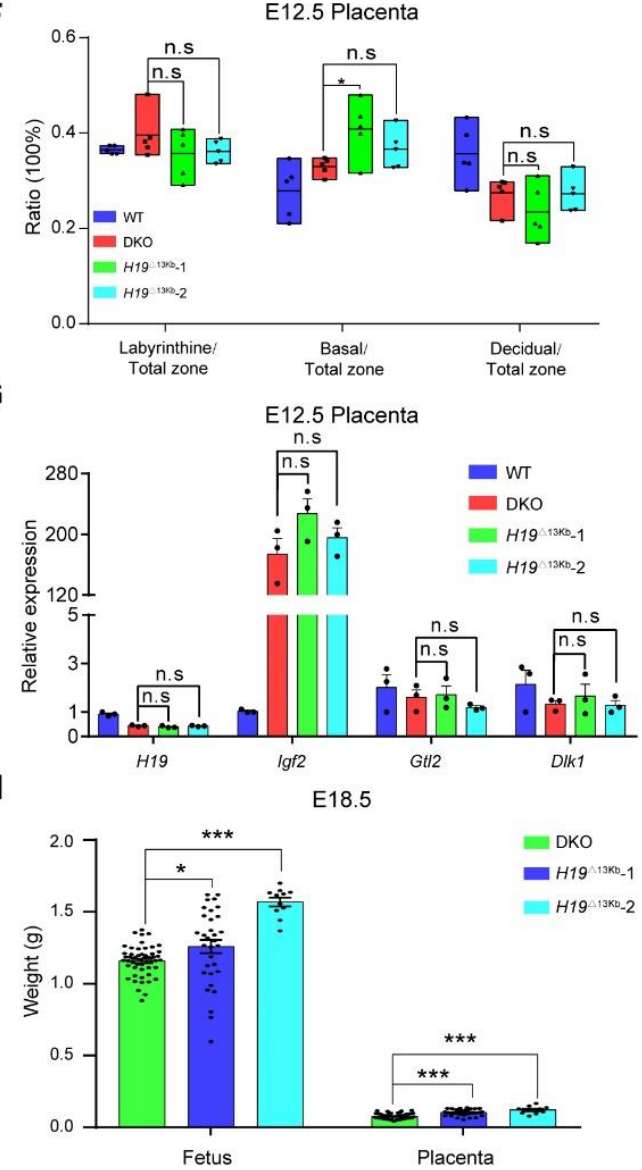

K

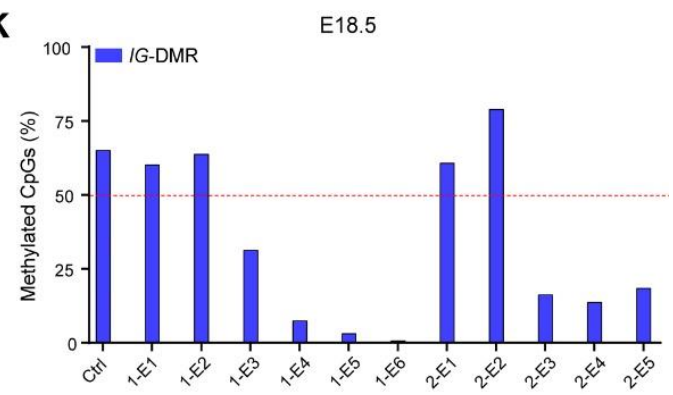

Figure 5. $H 1^{\Delta}{ }^{13 \mathrm{~kb}}$ rescues the abnormal development of SC embryos at mid-gestation.

(A) Schematic diagram of sgRNAs targeting for the removal of H19-DMR and $H 19$ transcription unit (about 13-kb).

(B) The ratios of E12.5 normal and abnormal SC embryos in transferred 2-cell embryos derived from WT ( $n=17$ recipients), DKO ( $n=11$ recipients) and $H 19 \Delta^{13 \mathrm{~kb}}\left(n=6\right.$ recipients for $H 19 \Delta^{13 \mathrm{~kb}}$ 1 and $n=7$ for $H 19^{\triangle 13 \mathrm{~kb}}-2$ ) AG-haESCs. Numbers under the bars indicate E12.5 
embryos/transferred two-cell embryos.

(C) The fetal and placental weights of E12.5 SC embryos derived from WT $(n=25)$, DKO $(n=$ 36) and $H 1{ }^{13 \mathrm{~kb}}$ ( $n=27$ for cell line1 and $n=25$ for cell line2) AG-haESCs, showing comparable developmental potential of $H 19^{\triangle 13 \mathrm{~kb}}$ and DKO SC embryos.

(D) Images of $H 19^{\triangle 13 \mathrm{~kb}} \mathrm{SC}$ fetuses (E12.5) showing normal development. White arrows show the umbilical cords. Experiments were repeated five times independently, with similar results.

(E) Histological sections of the placentas from $H 19^{\triangle 13 \mathrm{~kb}} \mathrm{SC}$ fetus at day 12.5 gestation. Gly C, glycogen cells; ST, spongiotrophoblast. Experiments were repeated five times independently, with similar results.

(F) The ratios of labyrinthine zone, basal zone and decidual zone to total placenta areas of WT, DKO and $H 19^{\triangle 13 \mathrm{~kb}}$ placentas (E12.5), indicating comparable development of DKO and H19 ${ }^{\triangle 13 \mathrm{~kb}}$ placentas. $n=5$ placentas for each group and at least 5 sections per placenta were analyzed.

Middle lines are shown as the mean.

(G) Transcriptional analysis of $H 19$, Igf2, Gtl2 and Dlk1 genes in WT, DKO and $H 19 \triangle 13 \mathrm{~kb}$ placentas ( $n=3$ placentas for each group). The expression values were normalized to that of Gapdh.

(H) The ratios of normal and abnormal SC embryos (E18.5) in transferred 2-cell embryos derived from WT ( $n=8$ recipients), DKO ( $n=9$ recipients) and $H 19^{\Delta 13 \mathrm{~kb}}\left(n=11\right.$ recipients for $H 19^{\Delta 13 \mathrm{~kb}-1}$

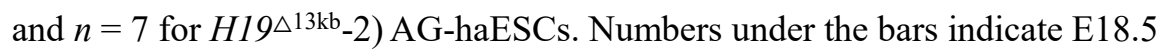

embryos/transferred two-cell embryos.

(I) The fetal and placental weights of E18.5 SC embryos derived from DKO $(n=51)$ and $H 19 \triangle 13 \mathrm{~kb}$ ( $n=32$ for $H 19^{\triangle 13 \mathrm{~kb}}-1$ and $n=11$ for $H 19^{\triangle 13 \mathrm{~kb}}-2$ ) AG-haESCs, showing highly developmental potential of $H 19 \triangle 13 \mathrm{~kb}$ compared to DKO SC embryos. Data are shown as the average mean \pm s.e.m in B, C and G-I. $* P<0.05,{ }^{* *} P<0.01,{ }^{* * *} P<0.001$. n.s means no significant difference.

$(\mathrm{J}$ and $\mathrm{K})$ Images of $H 19^{\triangle 13 \mathrm{~kb}} \mathrm{SC}$ embryos on E18.5 (J) and their methylation state in the $I G$-DMR (K) The black dotted box shows the retard embryo and red asterisks show degenerative embryos. The dotted line in $(\mathrm{K})$ shows theoretical value of methylation $(50 \%)$. 
A

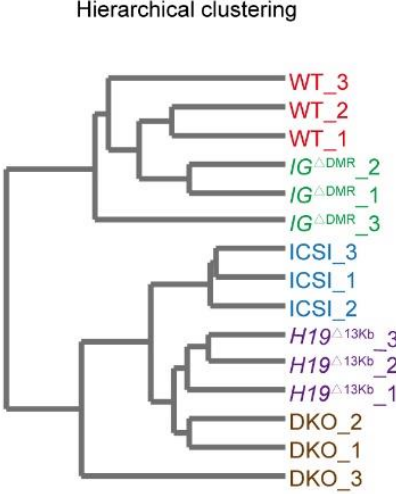

B

D

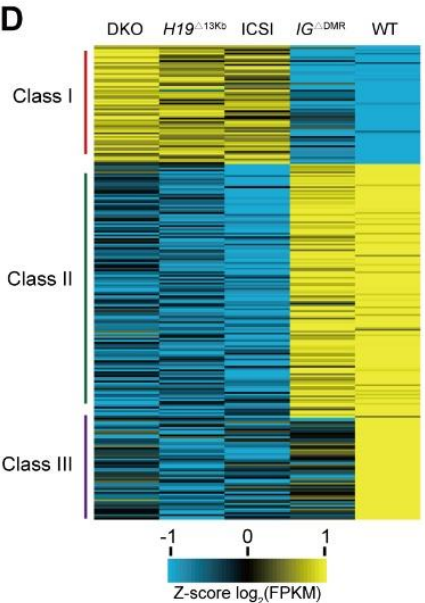

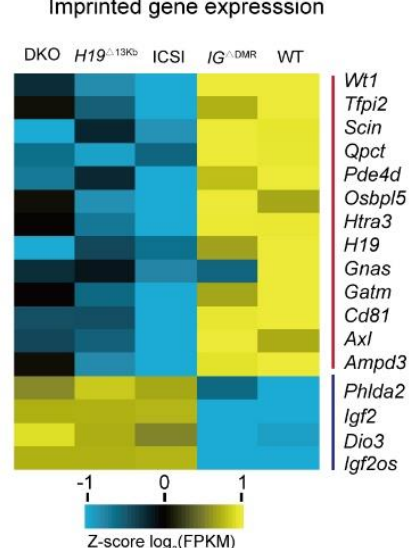

E

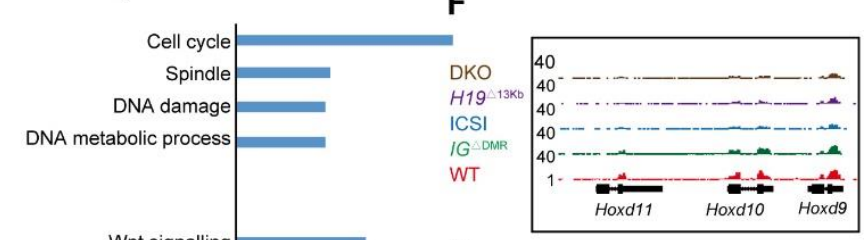

Class II

Skeletal system development Embryonic limb morphogenesis

Class III

\section{C}
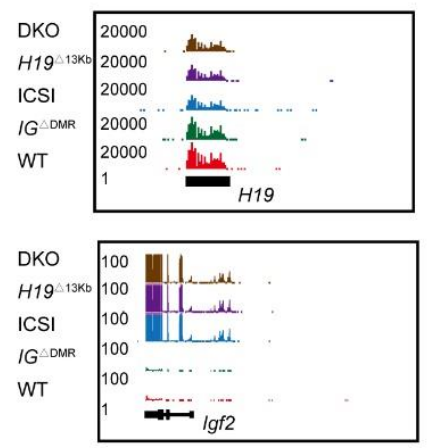

G

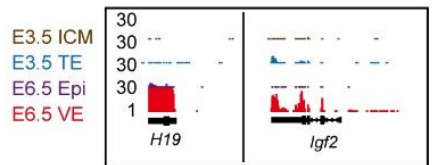

Figure 6. Global transcriptome analysis of SC placentas with different deletions.

(A) The hierarchical clustering analysis of RNA dataset from placentas obtained through oocyte injection of sperm, WT, $I G^{\triangle \mathrm{DMR}}, H 19^{\triangle 13 \mathrm{~kb}}$ and DKO AG-haESCs respectively.

(B) Differentially expressed imprinted genes in different placentas. Representative genes enriched in each cluster are shown in the right column.

(C) UCSC Genome Browser (University of California, Santa Cruz) snapshots of $H 19$ and Igf2 expression levels in different placentas.

(D and E) The heatmap showing 3 classes of differentially expressed genes among different placentas (D) and Gene Ontology (GO) analysis of genes in the 3 classes (E).

(F) Genome Browser snapshots of Hoxd11, Hoxd10 and Hoxd9 expression levels in different placentas.

(G) Genome Browser snapshots of $H 19$ and $\operatorname{Ig} f 2$ expression levels in E3.5 and E6.5 embryos, according to previous data (Zhang et al., 2018). 
A

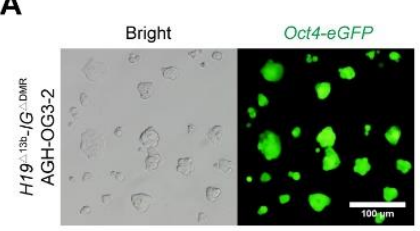

D

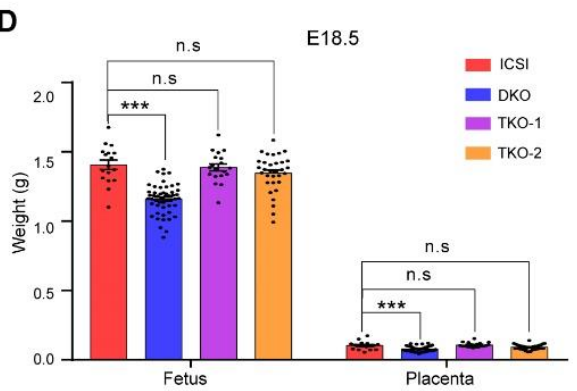

$\mathbf{F}$

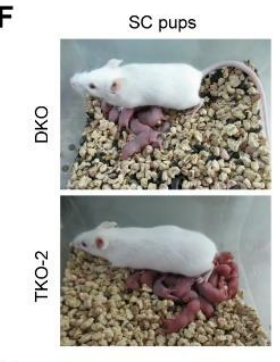

$\mathbf{J}$

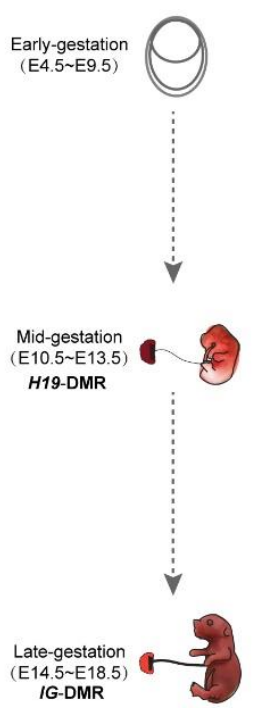

G

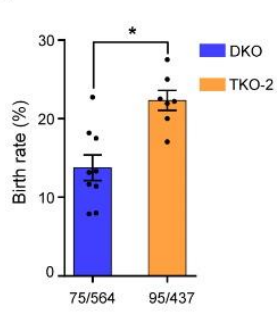

WT
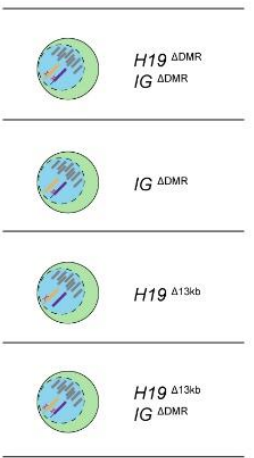

B

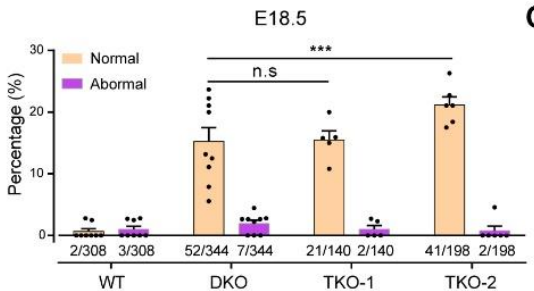

E
C

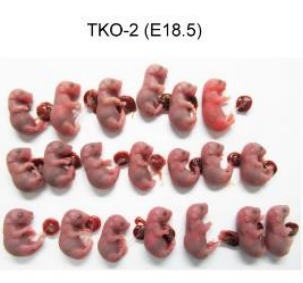

$\lg 2$

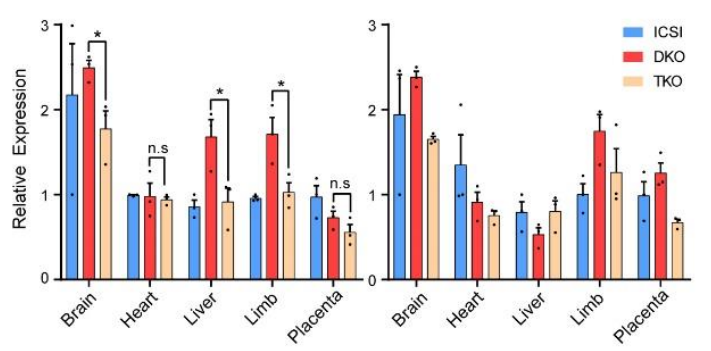

I
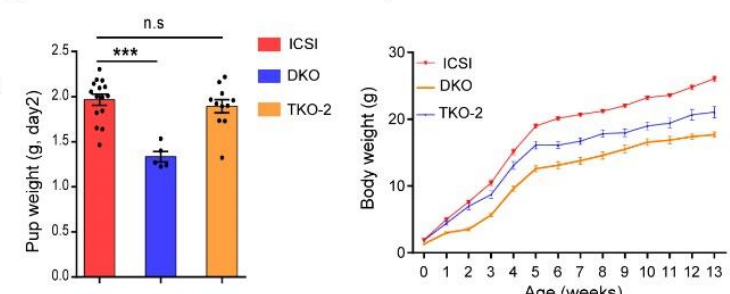

Age (weeks)

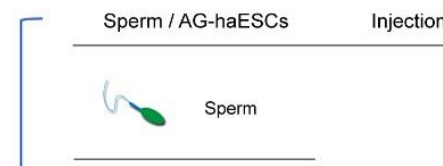

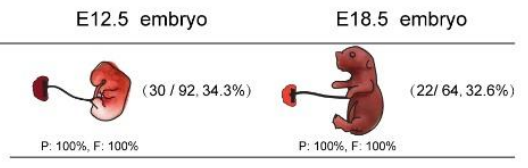
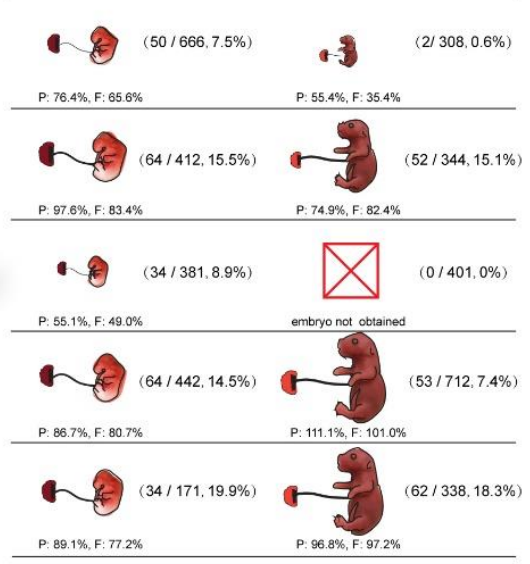

Figure 7. Combined deletions of H19, H19-DMR and IG-DMR in AG-haESCs further increases the prenatal and postnatal development of $\mathrm{SC}$ embryos.

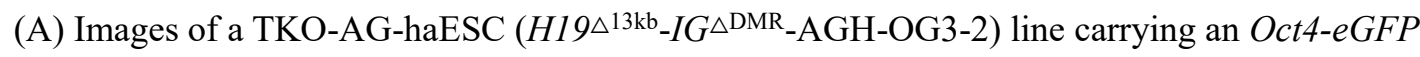
transgene.

(B) The ratios of normal and abnormal E18.5 SC embryos in transferred 2-cell SC embryos derived from WT ( $n=8$ recipients), DKO ( $n=9$ recipients) and TKO $(n=5$ recipients for TKO- 1 and 6 for TKO-2) AG-haESCs. Numbers under the bars indicate E18.5 embryos/transferred twocell embryos.

(C) Images of E18.5 TKO SC embryos derived from TKO-2 cells. Experiments were repeated five times independently, with similar results. 
(D) The fetal and placental weights of E18.5 embryos derived from sperm $(n=17)$, DKO $(n=51)$ and TKO ( $n=19$ for TKO-1 and $n=32$ for TKO-2) AG-haESCs, showing comparable development of TKO SC and control embryos (ICSI).

(E) Transcriptional analysis of imprinted genes $H 19$ and $\operatorname{Ig} 2 \mathrm{2}$ in different tissues of E18.5 fetuses (ICSI, DKO and TKO), including brain, heart, liver, limb and placenta $(n=3$ samples for each group). The expression values were normalized to that of Gapdh.

(F and G) The birth rates of SC pups from ICAHCI experiments using DKO and TKO-2 cells (G). Representative images of recipient mouse and SC pups shown in (F).

(H) Higher body weight of TKO-2 SC pups than that DKO pups on day 2 after birth.

(I) Growth curve of ICSI mice (females, $n=9)$, DKO $(n=4)$ and TKO-2 SC mice $(n=10)$, showing that TKO further improve the postnatal developmental potential of SC mice. Data are shown as the average mean \pm s.e.m in B, D, E and G-I. ${ }^{*} P<0.05$, ${ }^{* * *} P<0.001$. n.s means no significant difference.

(J) Summary of the temporal regulation of paternal genomic imprinting (H19-DMR and IG-DMR) during SC embryonic development. The percentages of placental $(\mathrm{P})$ and fetal $(\mathrm{F})$ weights are based on the placental and fetal weights of ICSI. The numerator in parentheses means the alive embryos obtained and denominator means the total number of transferred two-cell embryos. 


\section{SUPPLEMENTAL FIGURES}

A

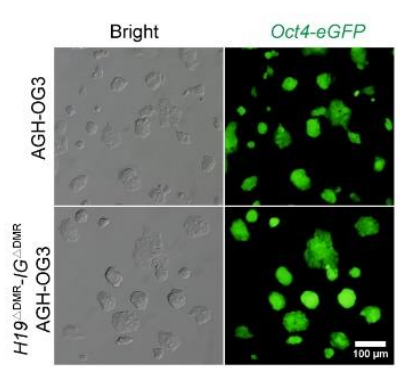

C

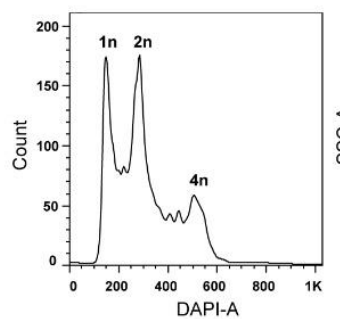

D

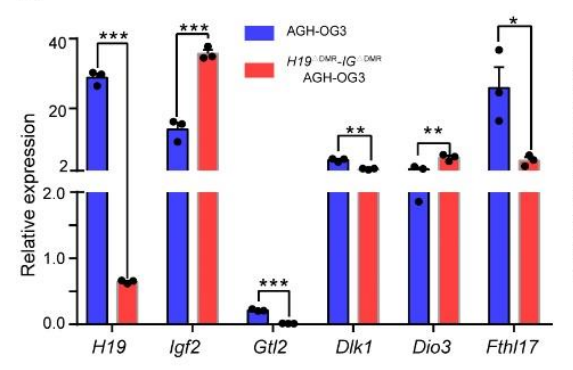

B
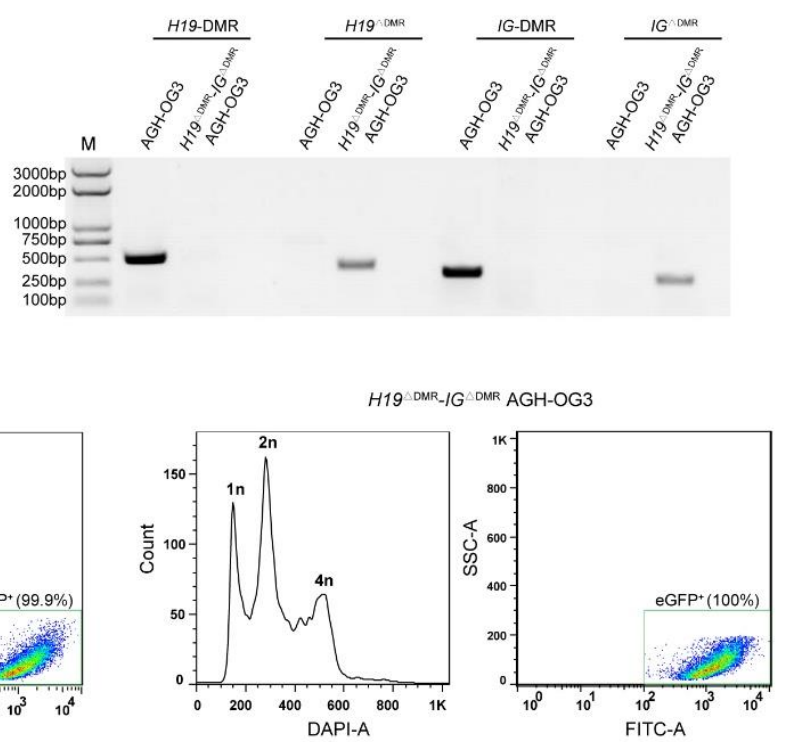

E

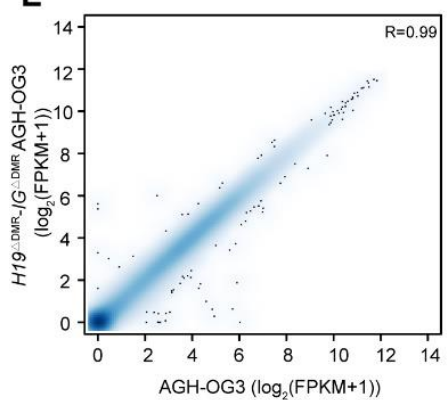

$F$

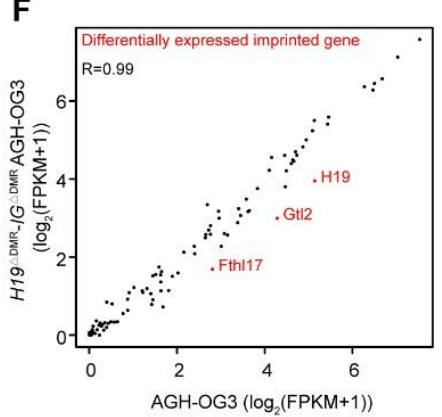

G
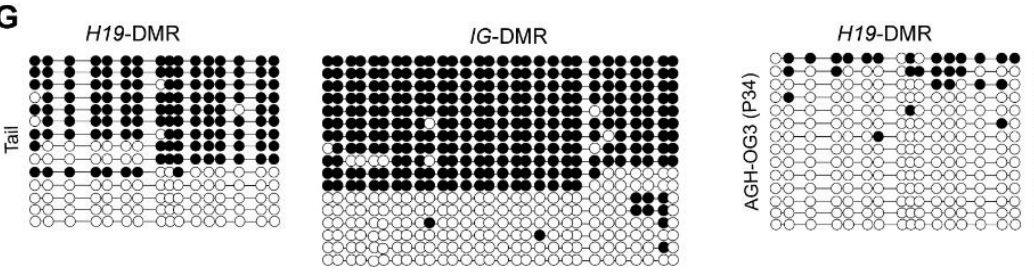

IG-DMR

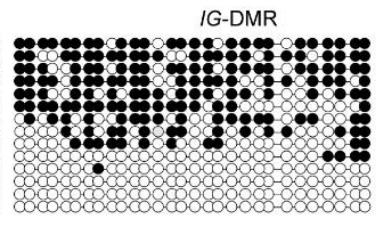

Figure S1. Characterizations of WT and DKO AG-haESCs. Related to Figure 1.

(A) Images of WT AG-haESC (AGH-OG3) and DKO-AG-haESC ( 19 $\left.^{\triangle} \triangle \mathrm{DMR}_{-} I G^{\triangle \mathrm{DMR}}-\mathrm{AGH}-\mathrm{OG} 3\right)$ lines carrying an Oct4-eGFP transgene. Experiments were repeated three times independently, with similar results.

(B) Genotyping analysis of $H 19^{\triangle \mathrm{DMR}_{-}}-\mathrm{G}^{\triangle \mathrm{DMR}}$-AGH-OG3 (DKO-AG-haESC) lines, AGH-OG3

(WT-AG-haESCs) as the control sample. M, DNA marker.

(C) FACS-mediated haploid cell-enrichment.

(D) Transcriptional analysis of imprinted genes, H19, Igf2, Gtl2, Dlk1, Dio3 and Fthl17 in AGH-

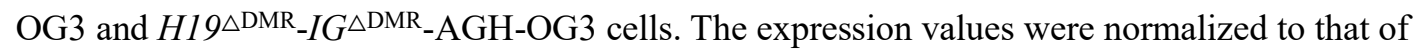
Gapdh. Data are shown as the average mean \pm s.e.m. $* P<0.05$, ${ }^{* *} P<0.01$, $* * * P<0.001$.

(E) Genome-scale transcriptional similarity between WT and DKO AG-haESCs. The gene expression level was measured as $\log _{2}(\mathrm{FPKM}+1)$. The Pearson correlation coefficient $(\mathrm{R})$ is 0.99 . (F) The scatter plot of imprinted gene expression from WT-AG-haESC and DKO-AG-haESCs. The gene expression level was measured as $\log _{2}(\mathrm{FPKM}+1)$. The Pearson correlation coefficient 
(R) is 0.99 .

(G Methylation analysis of the $H 19$ and $I G$-DMRs in tail (C57BL/6 female) and AGH-OG3. Open circles represent unmethylated $\mathrm{CpG}$ sites, whereas filled circles represent methylated $\mathrm{CpG}$ sites.

A

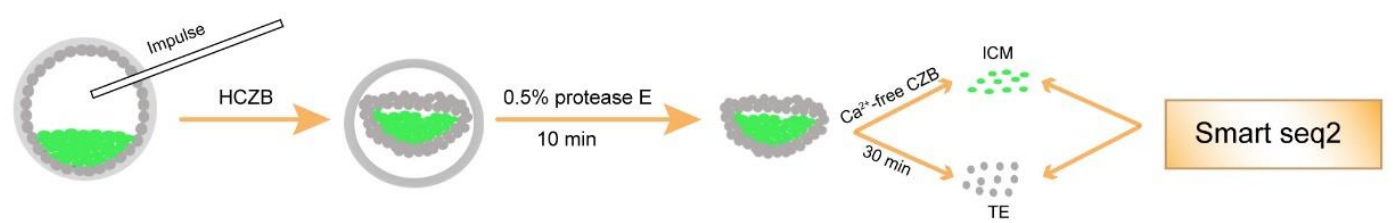

B

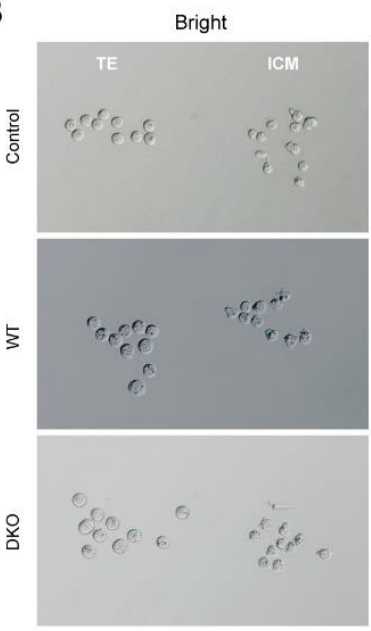

C

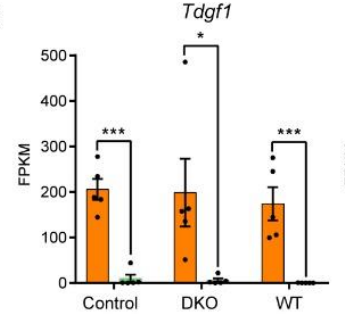

Oct4-eGFP

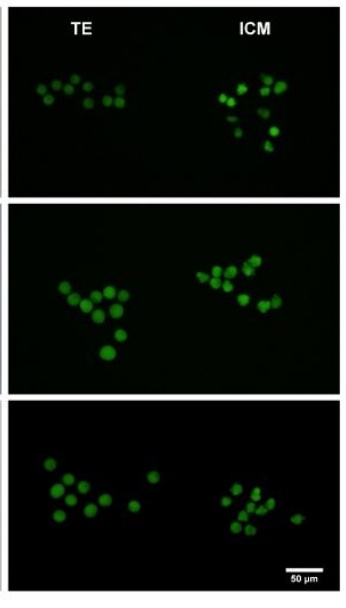

Esrrb

D

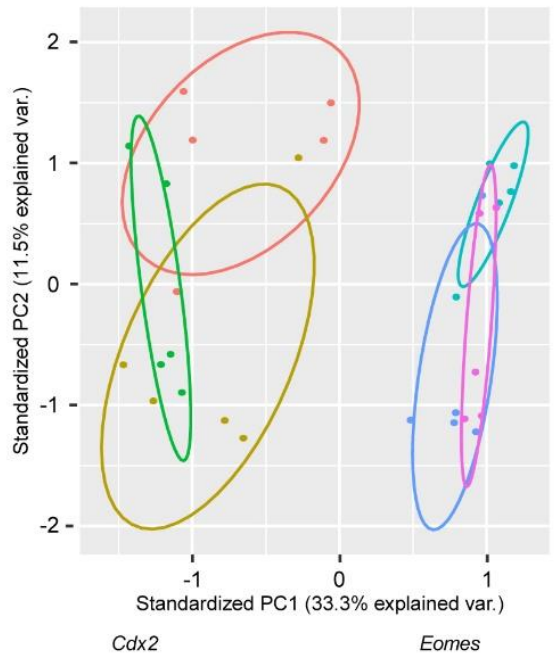

$\rightarrow$ C-ICM

$\rightarrow$ D-ICM

$\rightarrow$ W-ICM

$\rightarrow$ C-TE

$\rightarrow$ D-TE
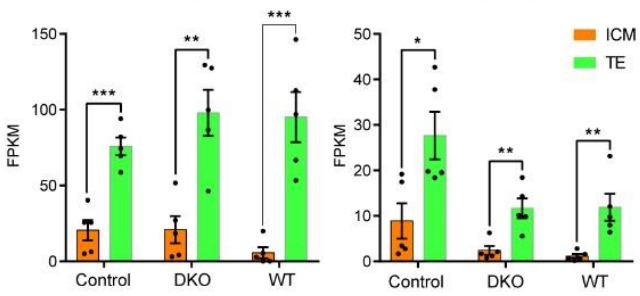

Figure S2. RNA-seq analysis of SC blastocysts. Related to Figure 1.

(A) Workflow of isolation of inner cell mass (ICM) and trophectoderm cell (TE) and RNA sequencing through Smart-seq2.

(B) Images showing that TE and ICM cells isolated from control (ICSI), WT and DKO SC blastocysts both carrying $e G F P$ signals. TE cells are big and smooth; ICM cells are small and outof-shape.

(C) The bar plot showing the expression level of ICM marker genes (Tdgfl and Esrrb) and TE marker genes ( $C d x 2$ and Eomes) in the isolated ICM and TE based on RNA-seq data. Data are shown as the average mean \pm s.e.m. ${ }^{*} P<0.05,{ }^{* *} P<0.01,{ }^{* * *} P<0.001$.

(D) The principle component analysis (PCA) of RNA-seq data from ICM and TE obtained through oocyte injection of sperm (C), WT (W) and DKO (D) AG-ESCs respectively. 
A

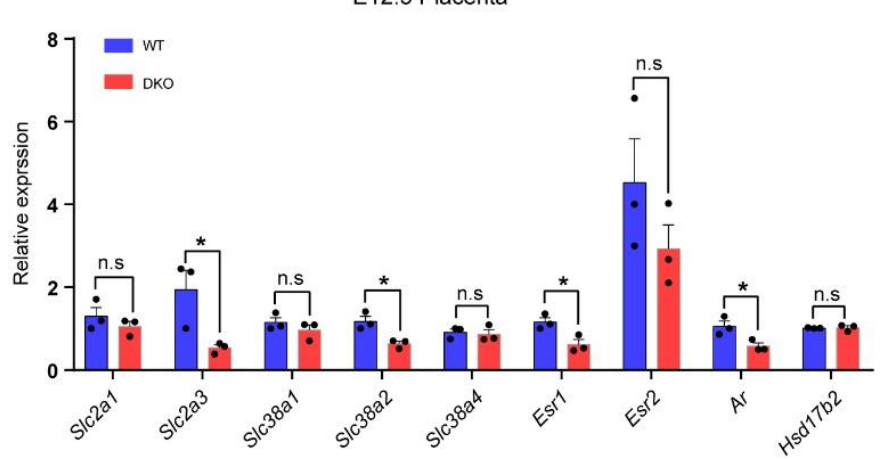

D

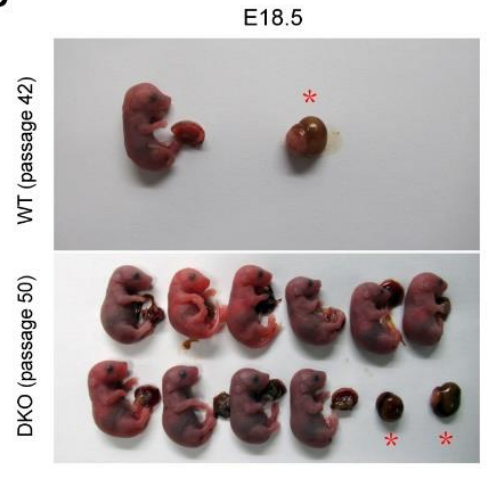

B

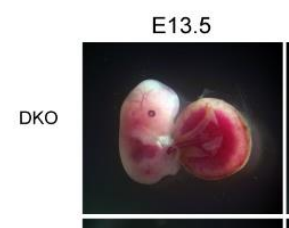

WT
(live)

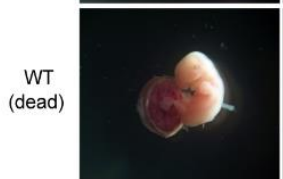

C

\begin{tabular}{|c|c|c|c|c|c|}
\hline \multirow{2}{*}{ Cell lines } & E11.5 & E12.5 & E13.5 & E14.5 & E15.5 \\
\hline & \multicolumn{4}{|c|}{ No. of dead embryos / No. of total embryos } & \\
\hline WT & $3 / 17$ & $15 / 50$ & 9/26 & $9 / 15$ & $13 / 22$ \\
\hline DKO & $0 / 18$ & $3 / 64$ & $0 / 18$ & $1 / 19$ & $0 / 31$ \\
\hline
\end{tabular}

Figure S3. H19-DMR and IG-DMR deletions (DKO) enhance the SC embryonic development. Related to Figure 3.

(A) Transcriptional analysis of glucose transporter genes (Slc2a1 and Slc2a3), system A amino acid transporter genes (Slc38a1, Slc38a2, and Slc38a4), estrogen receptor (Esr1 and Esr2), androgen receptor $(A r)$ and the inactivator of testosterone and estrogen (Hsd17b2) in E12.5 placentas of WT and DKO ( $n=3$ placentas for each group).

(B) Representative images of SC fetuses derived from DKO (top) and WT (middle and bottom) haploid cells. Experiments were repeated five times independently, with similar results.

(C) Summary of the number of dead embryos (E11.5 to E15.5) in all obtained SC embryos derived from WT and DKO AG-haESCs. Numerator means the dead embryos and denominator means the total number of embryos.

(D) Images of SC embryos on E18.5. Red asterisks show degenerative embryos.

(E) The ratios of normal and abnormal SC embryos (E18.5) derived from WT ( $n=8$ recipients)

and DKO ( $n=9$ recipients) haploid cells. Numbers under the bars indicate E18.5

embryos/transferred two-cell embryos. Data are shown as the average mean \pm s.e.m in A and E. ${ }^{*} P$ $<0.05$. n.s means no significant difference. 
A

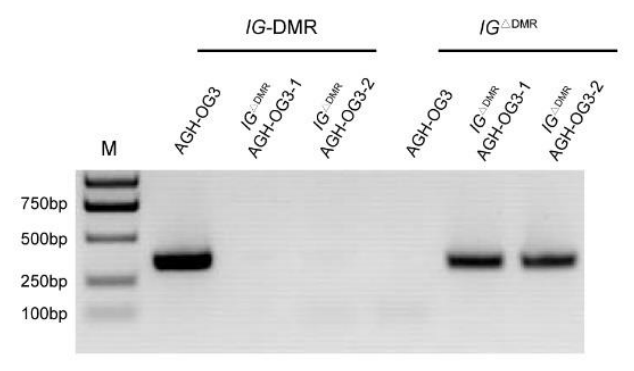

C

D

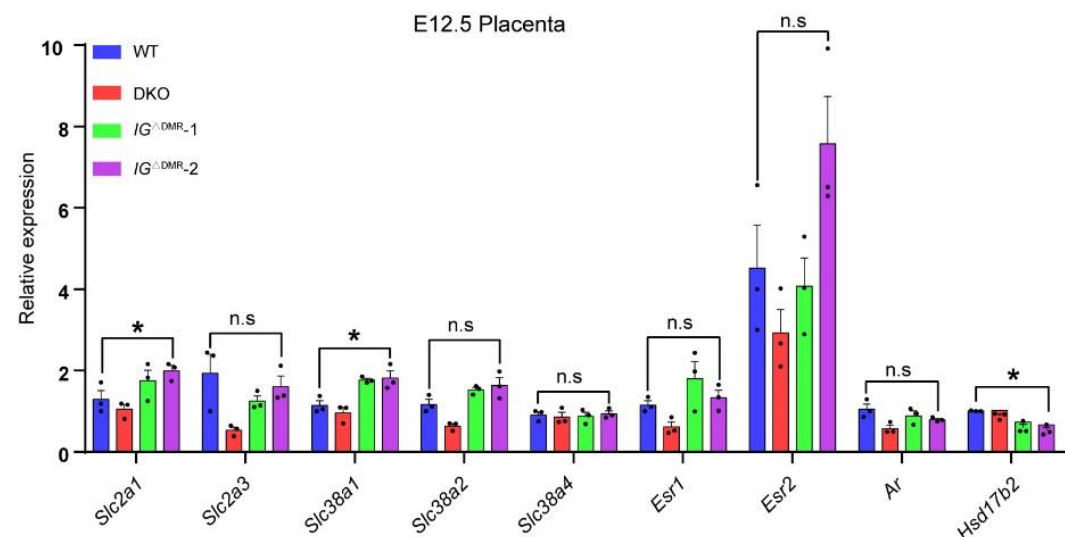

B

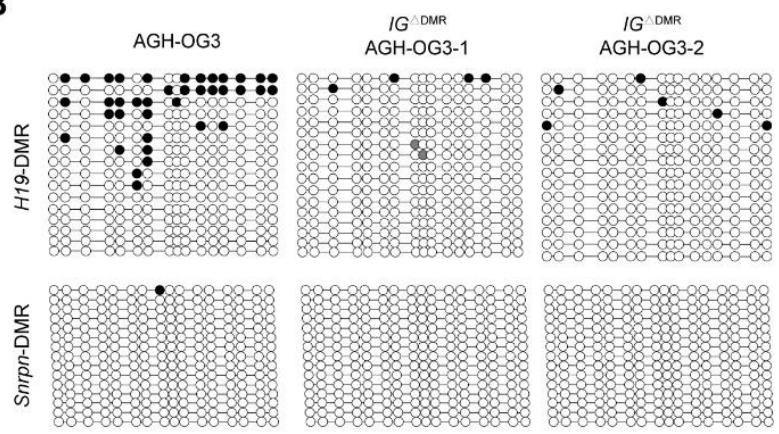

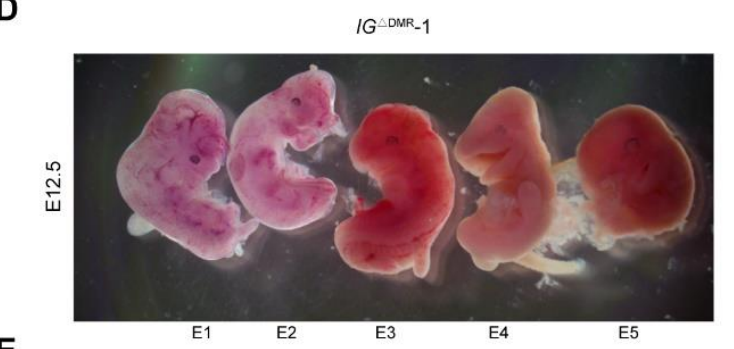

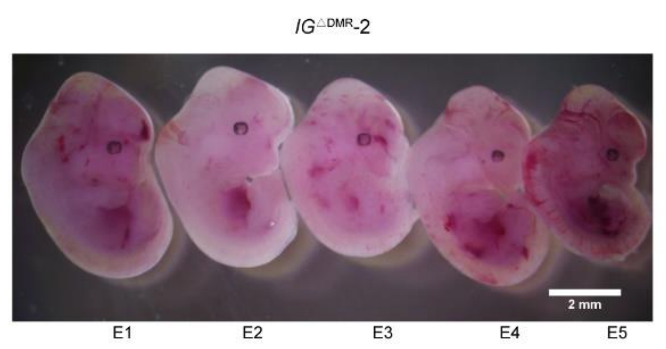

$\mathbf{E}$
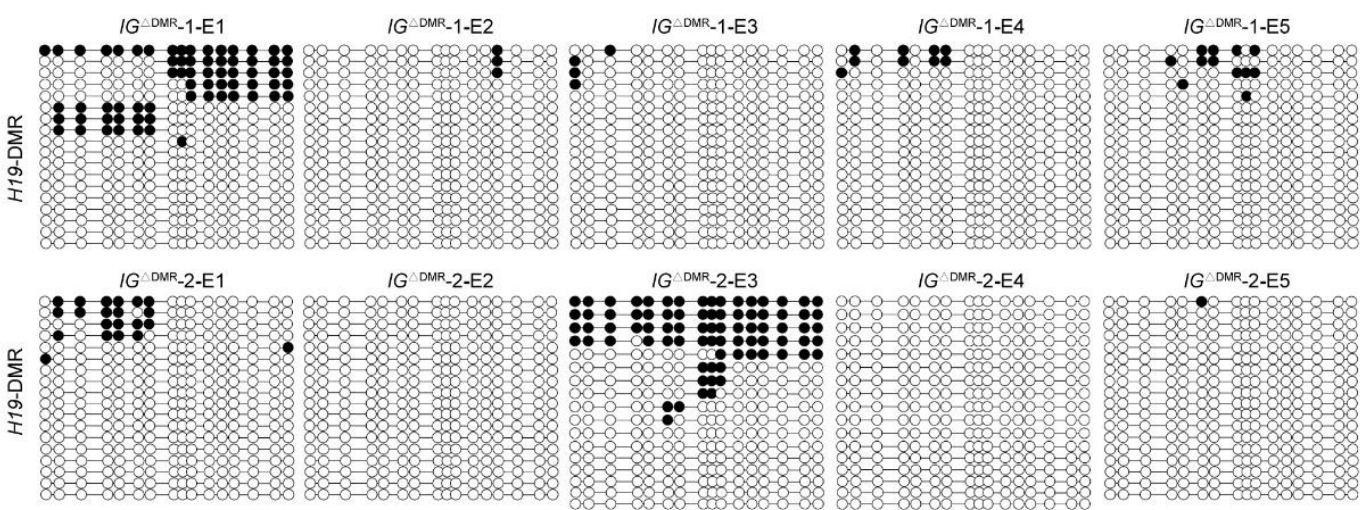

Figure S4. Abnormal development of SC embryos derived from $I G{ }^{\triangle D^{D M}}$-AG-haESCs.

Related to Figure 4.

(A) Genotyping analysis of two $I G^{\triangle \mathrm{DMR}}$-AGH-OG3 cell lines.

(B) Methylation analysis of the $H 19$ and Snrpn-DMRs in AGH-OG3 and $I G^{\triangle \mathrm{DMR}}$-AGH-OG3.

Open circles represent unmethylated $\mathrm{CpG}$ sites, whereas filled circles represent methylated $\mathrm{CpG}$ sites.

(C) Transcriptional analysis of glucose transporter genes (Slc2a1 and Slc2a3), system A amino acid transporter genes (Slc38a1, Slc38a2, and Slc38a4), estrogen receptor (Esr1 and Esr2), 
androgen receptor $(\mathrm{Ar})$ and the inactivator of testosterone and estrogen (Hsd17b2) in E12.5 placentas of WT, DKO and $I G^{\triangle \mathrm{DMR}}$ ( $n=3$ placentas for each group). Data are shown as the average mean \pm s.e.m. ${ }^{*} P<0.05$. n.s means no significant difference.

(D and E) Representative mages of $I G^{\triangle \mathrm{DMR}} \mathrm{SC}$ embryos on E12.5 (D) and methylation analysis of the H19-DMR in these embryos (E). Open circles represent unmethylated CpG sites, whereas filled circles represent methylated $\mathrm{CpG}$ sites.

A

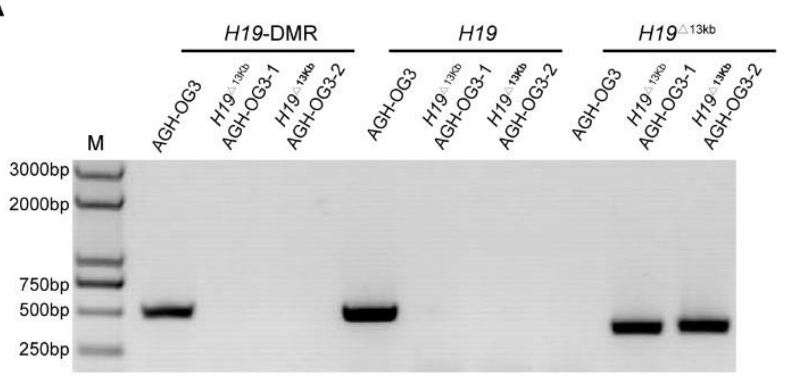

B

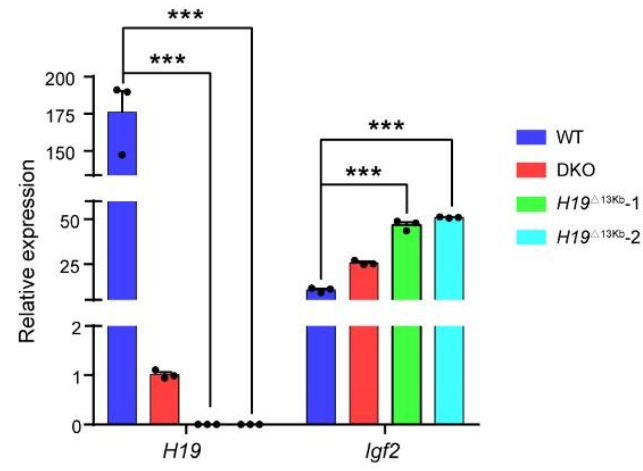

C

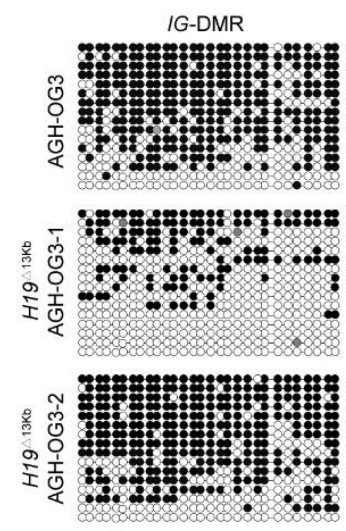

D

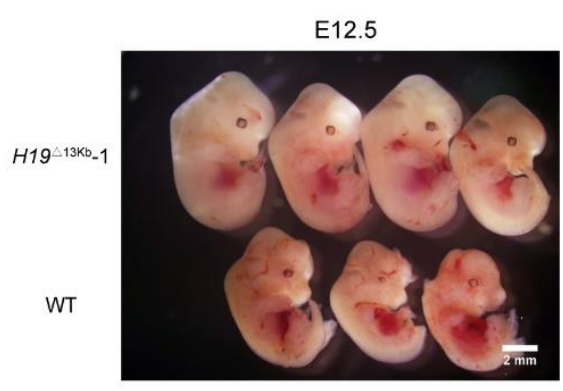

E

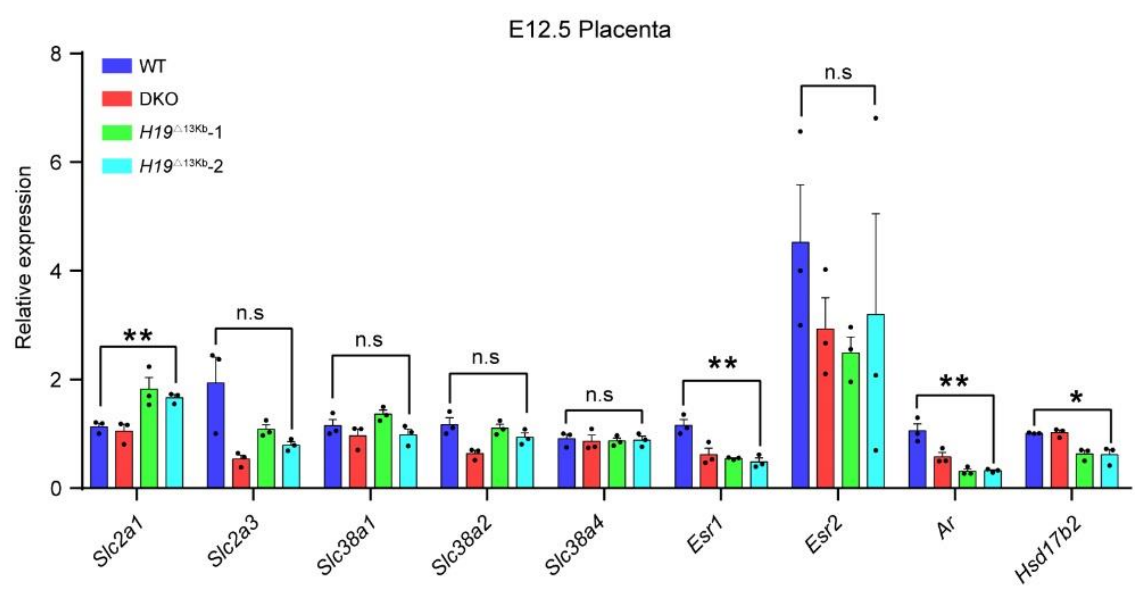

Figure S5. Characterizations of $\boldsymbol{H 1}{ }^{\Delta}{ }^{13 k b}$-AG-haESCs. Related to Figure 5.

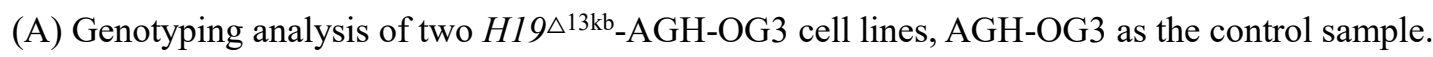
M, DNA marker.

(B) Transcriptional analysis of $H 19$ and $I g f 2$ genes in AGH-OG3, $H 1{ }^{\Delta{ }^{\mathrm{DMR}}}{ }_{-} I G^{\triangle \mathrm{DMR}_{-}}{ }_{-} \mathrm{AGH}-\mathrm{OG} 3$ and $H 19^{\triangle 13 \mathrm{~kb}-A G H-O G 3}$ cells, showing no $H 19$ expression in $H 19^{\triangle 13 \mathrm{~kb}}$ AG-haESCs. The expression values were normalized to that of Gapdh.

(C) Methylation analysis of the $I G$ and Snrpn-DMRs in $H 19^{\triangle 13 k b-A G H-O G 3}$ and AGH-OG3. 
Open circles represent unmethylated $\mathrm{CpG}$ sites, whereas filled circles represent methylated $\mathrm{CpG}$ sites.

(D) Images of $H 1{ }^{\triangle 13 \mathrm{~kb}}$ and WT SC embryos on E12.5. Experiments were repeated three times independently, with similar results.

(E) Transcriptional analysis of glucose transporter genes (Slc2al and Slc2a3), system A amino acid transporter genes (Slc38a1, Slc38a2, and Slc38a4), estrogen receptor (Esr1 and Esr2), androgen receptor $(A r)$ and the inactivator of testosterone and estrogen $(H s d 17 b 2)$ in E12.5 placentas of WT, DKO and $H 19^{\triangle 13 \mathrm{~kb}}$ ( $n=3$ placentas for each group). Data are shown as the average mean \pm s.e.m in B, E. ${ }^{*} P<0.05,{ }^{* *} P<0.01,{ }^{* * *} P<0.001$. n.s means no significant difference.
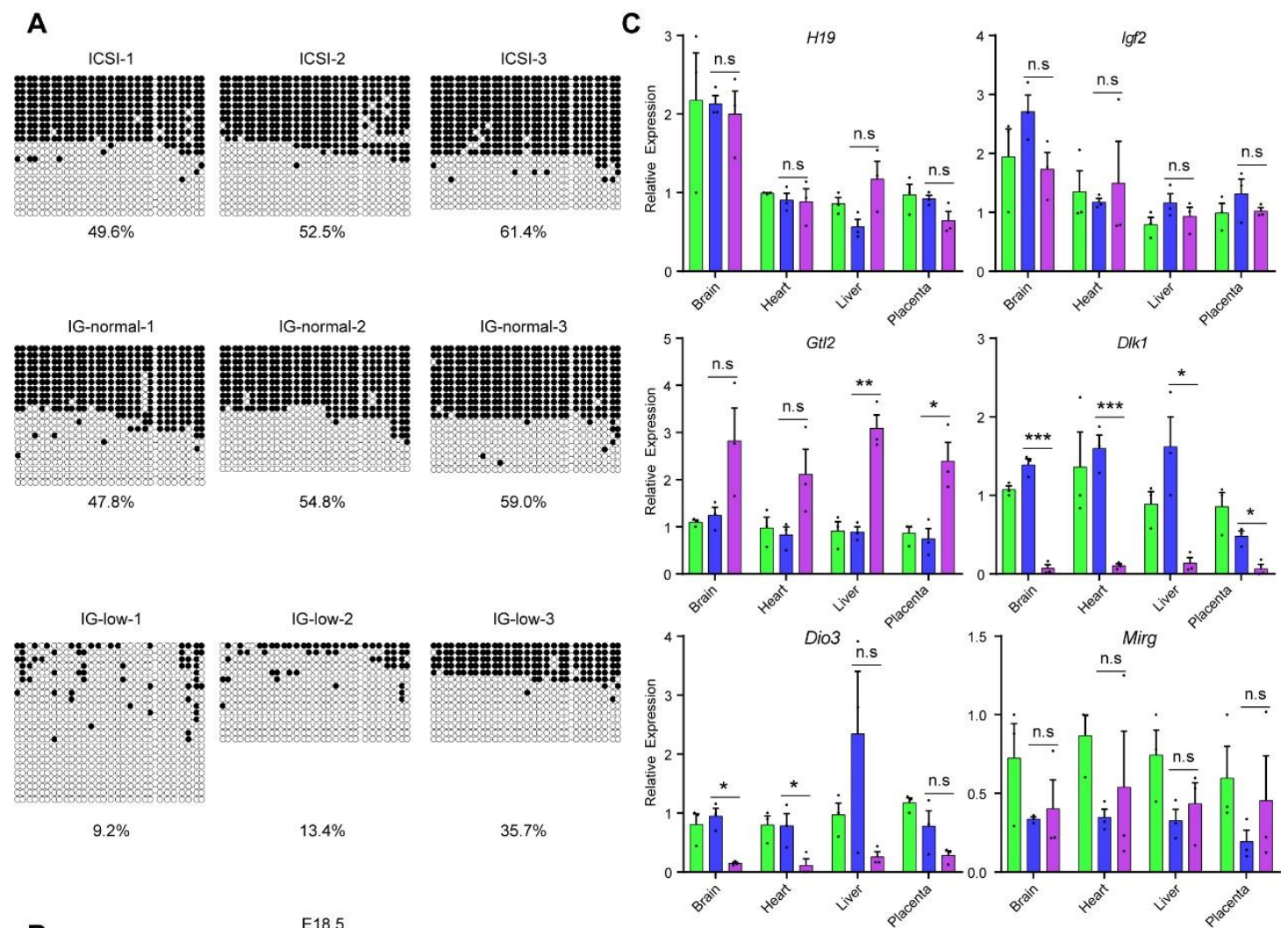

B
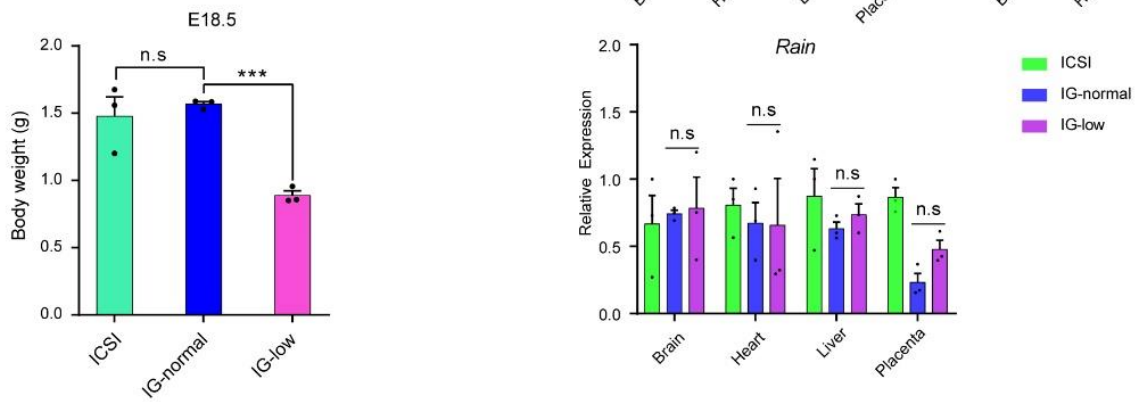

Figure S6. The correlation between hypomethylation of $I G$-DMR and growth retardation of SC embryos from $H_{19}{ }^{13 k b}$-AGH-OG3 cells at late-gestation. Related to Figure 5.

(A) Methylation analysis of IG-DMR on E18.5 embryos derived from sperm (ICSI) and $H 19^{\triangle 13 \mathrm{~kb}}$ AGH-OG3. $n=3$ embryos for each group. IG-normal SC embryos carrying normal methylation and IG-low SC embryos with hypomethylation compare with ICSI. Open circles represent 
unmethylated $\mathrm{CpG}$ sites, whereas filled circles represent methylated $\mathrm{CpG}$ sites.

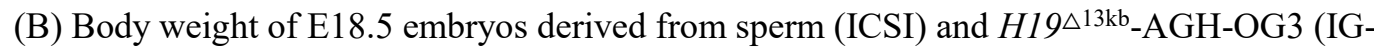
normal and IG-low), showing that IG-low embryos exhibit growth retardation.

(C) Transcriptional analysis of imprinted genes H19, Igf2, Gtl2, Dlk1, Dio3, Mirg and Rain in different tissues of E18.5 pups (ICSI, IG-normal and IG-low), including brain, heart, liver and placenta ( $n=3$ samples for each group). The expression values were normalized to that of Gapdh. Data are shown as the average mean \pm s.e.m in B and C. ${ }^{*} P<0.05, * * P<0.01, * * * P<0.001$. n.s means no significant difference.

\section{A}

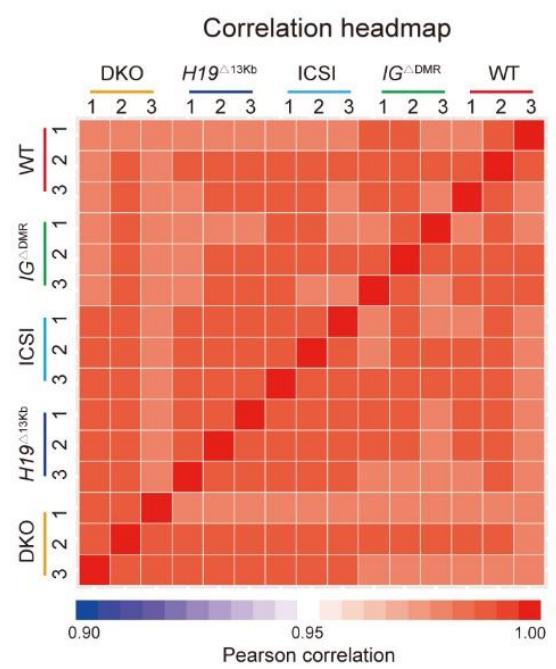

B

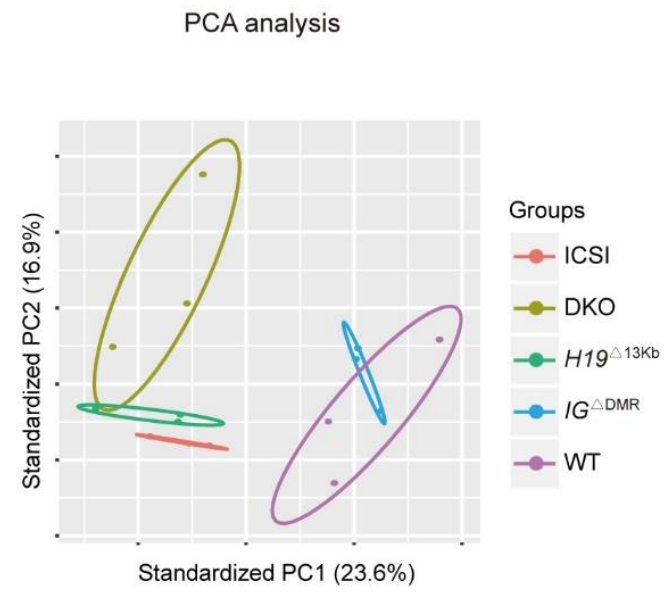

Figure S7. RNA-seq analysis of placentas with different deletions. Related to Figure 6.

(A) The correlation heatmap of RNA dataset from placentas obtained through oocyte injection of sperm, WT, DKO, $I G^{\triangle \mathrm{DMR}}$, and $H 19^{\triangle 13 \mathrm{~kb}}$ AG-haESCs respectively.

(B) The principle component analysis (PCA) based on all expressed genes of RNA-seq data. 
A
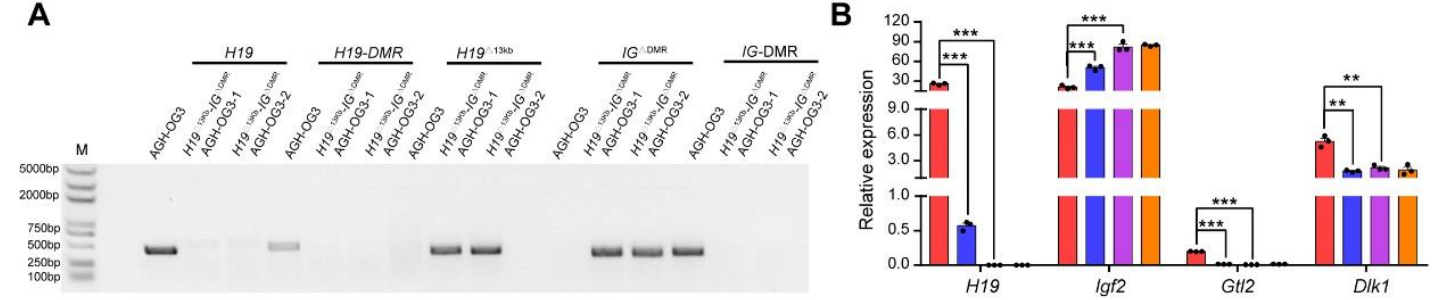

C
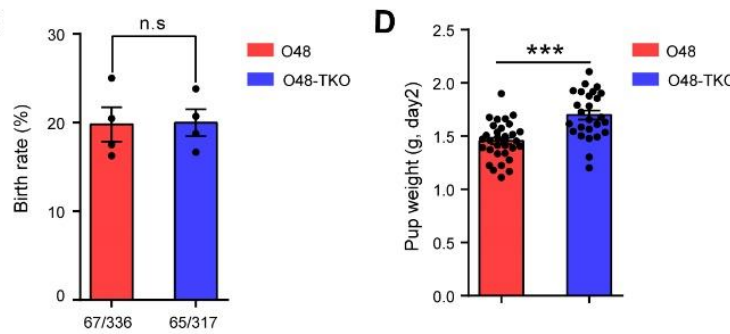

E
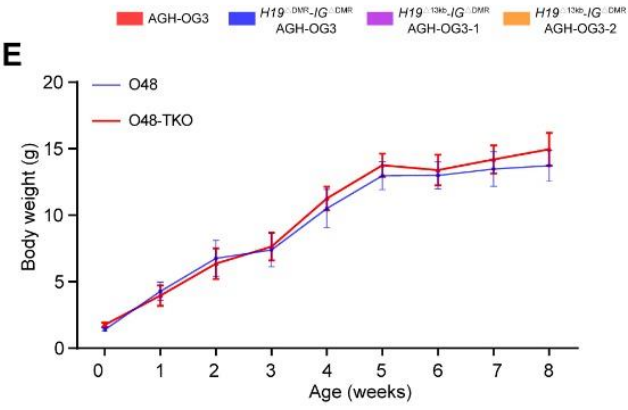

$\mathbf{F}$

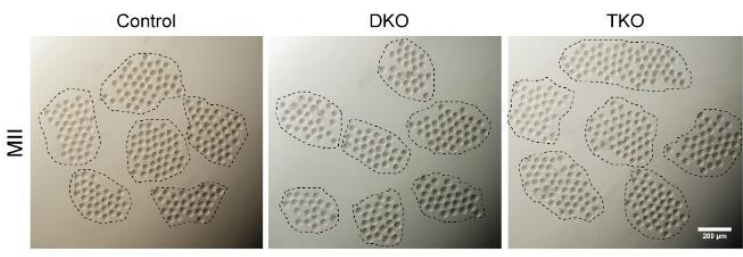

G

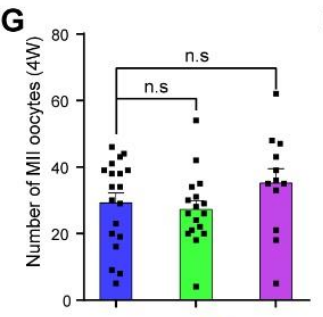

H

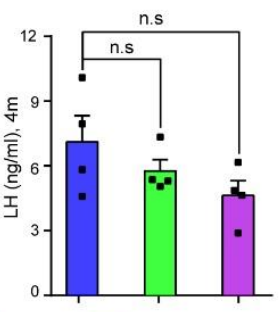

I

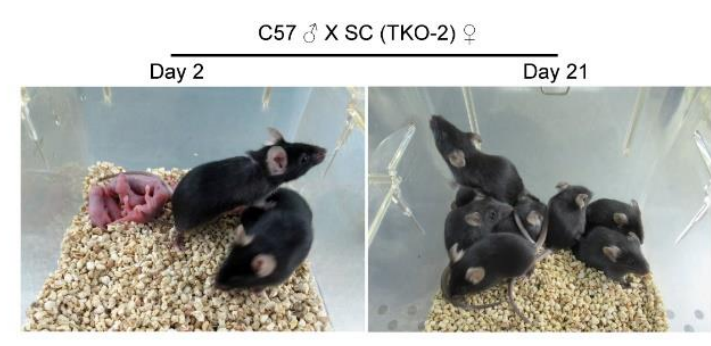

$\mathbf{J}$

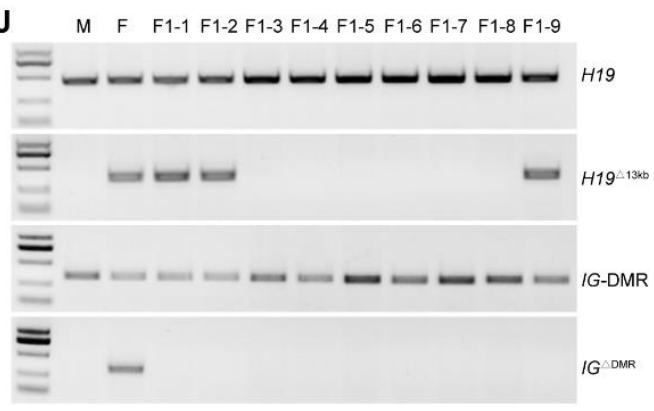

Figure S8. Characterizations of TKO AG-haESCs. Related to Figure 7.

(A) Genotyping analysis of two $H 19^{\triangle 13 \mathrm{~kb}}-I G^{\triangle \mathrm{DMR}}-\mathrm{AGH}-\mathrm{OG} 3$ cell lines (TKO-AG-haESCs), AGH-OG3 as the control sample. M, DNA marker.

(B) Transcriptional analysis of H19, Igf2, Gtl2 and Dlk1 genes in AGH-OG3, H19 $\triangle \mathrm{DMR}_{-} I G^{\triangle \mathrm{DMR}_{-}}$ AGH-OG3 and $H 19^{\triangle 13 \mathrm{~kb}}-I G^{\triangle \mathrm{DMR}}$-AGH-OG3 (two different cell lines). The expression values were normalized to that of Gapdh.

(C) The birth rate of newborn SC pups from ICAHCI of O48 and O48-TKO cells. The O48 cell line was described in previous work (termed also as $\left.H 19^{\triangle \mathrm{DMR}}-I G^{\triangle \mathrm{DMR}}-\mathrm{AGH}-2\right)$ (Zhong et al., 2015).

(D) Body weight of O48-TKO SC pups are significantly higher than that of O48 on day 2 after birth.

(E) Growth curve of O48 $(n=10)$ and O48-TKO SC mice $(n=11)$, showing comparable growth rate between DKO and TKO SC pups.

(F) Images of mature oocytes obtained from superovulated 4-week old females, including ICSI (control), DKO and TKO mice. The dotted lines indicate oocytes from one female.

(G) Quantification of mature oocytes derived from ICSI ( $n=19$ females), DKO ( $n=17$ females) 
and TKO ( $n=12$ females) groups.

(H) Measurement of luteinizing hormone (LH) in plasma from 4-month mice (ICSI, DKO and TKO). $n=4$ mice for each group. Data are shown as the average mean \pm s.e.m in B-D, G and H. ${ }^{* *} P<0.01,{ }^{* * *} P<0.001$. n.s means no significant difference.

(I) Adult TKO SC mice and their offspring. Experiments were repeated three times independently, with similar results.

(J) Genotyping analysis of the $H 19, H 19$-DMR and $I G$-DMR deletions in the alive progeny of SC TKO mice.

Table S1. List of primer and sgRNA sequence information, related to experimental procedures

\begin{tabular}{|c|c|c|}
\hline Name & Sequence $\left(5^{\prime}-3^{\prime}\right)$ & Application \\
\hline Gapdh-qPCR-F & САСТСТTССАССТTCGATGC & \multirow{29}{*}{ Realtime PCF } \\
\hline Gapdh-qPCR-R & CTCTTGCTCAGTGTCCTTGC & \\
\hline H19-qPCR-F & TGTAAACCTCTTTGGCAATGCTGCC & \\
\hline H19-qPCR-R & TATTGATGGACCCAGGACCTCTGGT & \\
\hline Igf2-qPCR-F & CTAAGACTTGGATCCCAGAACC & \\
\hline Igf2-qPCR-R & GTTCTTCTCCTTGGGTTCTTTC & \\
\hline Gt12-qPCR-F & TTGCACATTTCCTGTGGGAC & \\
\hline Gt12-qPCR-R & AAGCACCATGAGCCACTAGG & \\
\hline Dlk1-qPCR-F & ACTTGCGTGGACCTGGAGAA & \\
\hline Dlk1-qPCR-R & CTGTTGGTTGCGGCTACGAT & \\
\hline Rain-qPCR-F & CGTGTGTGTGTGTGTGTGGT & \\
\hline Rain-qPCR-R & GCCAAGGTCTCTACCAGCAG & \\
\hline Mirg-qPCR-F & GGCAAGGTCTAGGATGGACA & \\
\hline Mirg-qPCR-R & CGCCAGCTTCTGAATACTCC & \\
\hline Dio3-qPCR-F & CCCATGACACAGATGAGCAC & \\
\hline Dio3-qPCR-R & CCTGAGAGCAAGCCAAAAAC & \\
\hline Fthl17-qPCR-F & TCAAGCCATGGAATGTGCCT & \\
\hline Fthl17-qPCR-R & TGCTCCAGGAAATGGCAGAG & \\
\hline Slc38a1-qPCR-F & TCCTTCAGCCATAAAATCCCTC & \\
\hline Slc38a1-qPCR-R & CCACTCGTGTAGCCAAGATAC & \\
\hline Slc38a2-qPCR-F & CCGTCTGCCTTCTACATCAAG & \\
\hline Slc38a2-qPCR-R & CCGTCTGCCTTCTACATCAAG & \\
\hline Slc38a4-qPCR-F & CCTCGTGCCTACCATCAAATAC & \\
\hline Slc38a4-qPCR-R & AGACCAAAGCCCCAATCTTC & \\
\hline SIc2a1-qPCR-F & GATTGGTTCCTTCTCTGTCGG & \\
\hline SIc2a1-qPCR-R & CCCAGGATCAGCATCTCAAAG & \\
\hline SIc2a3-qPCR-F & CGCTTCTCATCTCCATTGTCC & \\
\hline SIc2a3-qPCR-R & TGAAGATAGTATTGACCACGCC & \\
\hline Esr1-qPCR-F & AACCGCCCATGATCTATTCTG & \\
\hline
\end{tabular}




\begin{tabular}{|c|c|c|}
\hline Esr1-qPCR-R & AGATTCAAGTCCCCAAAGCC & \\
\hline Esr2-qPCR-F & ACGAAGTAGGAATGGTCAAGTG & \\
\hline Esr2-qPCR-R & GGTTCTCTTGGCTTTGTTCAG & \\
\hline Ar-qPCR-F & CGACTATTACTTTCCACCCCAG & \\
\hline Ar-qPCR-R & TGCTGGCACATAGATACTTCTG & \\
\hline Hsd17b2 -qPCR-F & AGTTGTGACCATCAAACCCG & \\
\hline Hsd17b2 -qPCR-R & ATGAGCTTCTGTGTATGGACG & \\
\hline H19-BS-OF & GAGTATTTAGGAGGTATAAGAATT & \multirow{14}{*}{$\begin{array}{c}\text { Bisulfite } \\
\text { sequencing }\end{array}$} \\
\hline H19-BS-OR & ATCAAAAACTAACATAAAССССТ & \\
\hline H19-BS-IF & GTAAGGAGATTATGTTTATTTTTGG & \\
\hline H19-BS-IR & ССТСАТТААТСССАТААСТАТ & \\
\hline IG-BS-OF & TTAAGGTATTTTTTATTGATAAAATAATGTAGTTT & \\
\hline IG-BS-OR & ССТАСТСТАТААТАСССТАТАТААТТАТАССАТАА & \\
\hline IG-BS-IF2 & GATCTCGAGCTCAAGCTTCGCTATAAT & \\
\hline G-DU-II & TTATСАТАAАСАААТСССАТААСТTАСТ & \\
\hline \multirow{2}{*}{ IG-BS-IR2 } & CAGTTATCTAGATCCGGTGTTAGGAG & \\
\hline & TTAAGGAAAAGAAAGAAATAGTATAGT & \\
\hline Snrpn-BS-OF & TATGTAATATGATATAGTTTAGAAATTAG & \\
\hline Snrpn-BS-OR & AATAAACCCAAATCTAAAATATTTTAATC & \\
\hline Snrpn-BS-IF & AATTTGTGTGATGTTTGTAATTATTTGG & \\
\hline Snrpn-BS-IR & ATAАAАТАСАСТTТСАСТАСТААААТСС & \\
\hline IG-DMR-sgRAN1-F & CACCCGTACAGAGCTCCATGGCAC & \multirow{8}{*}{$\begin{array}{l}\text { sgRNAs for } \\
\text { deletion of } / G- \\
\text { DMR and } H 19- \\
\text { 13kb sequence }\end{array}$} \\
\hline IG-DMR-sgRAN1-R & AAACGTGCCATGGAGCTCTGTACG & \\
\hline IG-DMR-sgRNA2-F & CACCCTGCTTAGAGGTACTACGCT & \\
\hline IG-DMR-sgRNA2-R & AAACAGCGTAGTACCTCTAAGCAG & \\
\hline H19-13k-sgRNA1-F & CACCAGACCCGCCCAGGAAGGGAC & \\
\hline H19-13k-sgRNA1-R & AAACGTCCCTTCCTGGGCGGGTCT & \\
\hline H19-13k-sgRNA2-F & CACCAGAGTGCCATACGGAGACAG & \\
\hline H19-13k-sgRNA2-R & AAACCTGTCTCCGTATGGCACTCT & \\
\hline IG-DMR-wt-F & TGTATGGTACAAACCGGGTGA & \multirow{12}{*}{$\begin{array}{c}\text { Genotyping of } \\
\text { deleted H19- } \\
\text { DMR, IG-DMR } \\
\text { and H19-13kb by } \\
\text { CRISPR/Cas9 }\end{array}$} \\
\hline IG-DMR-wt-R & ACTTTTGCTGTGAGGCACTTG & \\
\hline IG-DMR-del-F & TGTGCAGCAGCAAAGCTAAG & \\
\hline IG-DMR-del-R & ATACGATACGGCAACCAACG & \\
\hline H19-wt-F & CACTGTATGCCCTAACCGCT & \\
\hline H19-wt-R & CATCACACCGGACCATGTCA & \\
\hline H19-DMR-wt-F & AGATGGGGTCATTCTTTTCC & \\
\hline H19-DMR-wt-R & ATTGCTCTTAGCTTCTGTTG & \\
\hline H19-DMR-del-F & GTGGTTAGTTCTATATGGGG & \\
\hline H19-DMR-del-R & TCTTACAGTCTGGTCTTGGT & \\
\hline H19-13kb-del-F & TGTGGGCTGAGTACTTGGAC & \\
\hline H19-13kb-del-R & GGGAGTTGGGATTAGTGTGA & \\
\hline
\end{tabular}




\section{REFERENCES}

Angiolini, E., Coan, P.M., Sandovici, I., Iwajomo, O.H., Peck, G., Burton, G.J., Sibley, C.P., Reik, W., Fowden, A.L., and Constancia, M. (2011). Developmental adaptations to increased fetal nutrient demand in mouse genetic models of Igf2-mediated overgrowth. FASEB journal : official publication of the Federation of American Societies for Experimental Biology 25, 1737-1745.

Arney, K.L. (2003). H19 and Igf2--enhancing the confusion? Trends Genet 19, 17-23.

Barlow, D.P., and Bartolomei, M.S. (2014). Genomic imprinting in mammals. Cold Spring Harb Perspect Biol 6.

Bartolomei, M.S., Zemel, S., and Tilghman, S.M. (1991). Parental imprinting of the mouse H19 gene. Nature 351, 153.

Bell, A.C., and Felsenfeld, G. (2000). Methylation of a CTCF-dependent boundary controls imprinted expression of the Igf2 gene. Nature 405, 482.

Bell, A.C., West, A.G., and Felsenfeld, G. (1999). The Protein CTCF Is Required for the Enhancer Blocking Activity of Vertebrate Insulators. Cell 98, 387-396.

Brunkow, M.E., and Tilghman, S.M. (1991). Ectopic expression of the H19 gene in mice causes prenatal lethality. Genes Dev 5, 1092-1101.

Burns, J.L., and Hassan, A.B. (2001). Cell survival and proliferation are modified by insulin-like growth factor 2 between days 9 and 10 of mouse gestation. Development 128, 3819 .

Chen, J., Suo, S., Tam, P.P., Han, J.J., Peng, G., and Jing, N. (2017). Spatial transcriptomic analysis of cryosectioned tissue samples with Geo-seq. Nature protocols 12, 566-580.

Choi, J., Huebner, A.J., Clement, K., Walsh, R.M., Savol, A., Lin, K., Gu, H., Di Stefano, B., Brumbaugh, J., Kim, S.Y., et al. (2017). Prolonged Mek1/2 suppression impairs the developmental potential of embryonic stem cells. Nature 548, 219-223.

Cleaton, M.A.M., Edwards, C.A., and Ferguson-Smith, A.C. (2014). Phenotypic Outcomes of Imprinted Gene Models in Mice: Elucidation of Pre- and Postnatal Functions of Imprinted Genes. Annual Review of Genomics and Human Genetics 15, 93-126.

Constancia, M., Angiolini, E., Sandovici, I., Smith, P., Smith, R., Kelsey, G., Dean, W., FergusonSmith, A., Sibley, C.P., Reik, W., et al. (2005). Adaptation of nutrient supply to fetal demand in the mouse involves interaction between the Igf2 gene and placental transporter systems. Proceedings of the National Academy of Sciences of the United States of America 102, 19219-19224.

Constância, M., Hemberger, M., Hughes, J., Dean, W., Ferguson-Smith, A., Fundele, R., Stewart, F., Kelsey, G., Fowden, A., Sibley, C., et al. (2002). Placental-specific IGF-II is a major modulator of placental and fetal growth. Nature 417, 945.

DeChiara, T.M., Efstratiadis, A., and Robertsen, E.J. (1990). A growth-deficiency phenotype in heterozygous mice carrying an insulin-like growth factor II gene disrupted by targeting. Nature 345 , 78.

DeChiara, T.M., Robertson, E.J., and Efstratiadis, A. (1991). Parental imprinting of the mouse insulinlike growth factor II gene. Cell 64, 849-859.

Eggenschwiler, J., Ludwig, T., Fisher, P., Leighton, P.A., Tilghman, S.M., and Efstratiadis, A. (1997). Mouse mutant embryos overexpressing IGF-II exhibit phenotypic features of the Beckwith-Wiedemann and Simpson-Golabi-Behmel syndromes. Genes Dev 11, 3128-3142.

Ferguson-Smith, A.C. (2011). Genomic imprinting: the emergence of an epigenetic paradigm. Nature reviews Genetics 12, 565-575. 
Graham, C.F. (1970). Parthenogenetic Mouse Blastocysts. Nature 226, 165.

Hark, A.T., Schoenherr, C.J., Katz, D.J., Ingram, R.S., Levorse, J.M., and Tilghman, S.M. (2000).

CTCF mediates methylation-sensitive enhancer-blocking activity at the H19/Igf2 locus. Nature 405, 486.

Jiang, J., Yan, M., Li, D., and Li, J. (2018). Genome tagging project: tag every protein in mice through 'artificial spermatids'. National Science Review nwy136.

Kaufman, M.H. (1973). Parthenogenesis in the mouse. Nature 242, 475-476.

Kawahara, M., Wu, Q., Takahashi, N., Morita, S., Yamada, K., Ito, M., Ferguson-Smith, A.C., and Kono, T. (2007). High-frequency generation of viable mice from engineered bi-maternal embryos. Nature biotechnology 25, 1045.

Kobayashi, S., Fujihara, Y., Mise, N., Kaseda, K., Abe, K., Ishino, F., and Okabe, M. (2010). The Xlinked imprinted gene family Fthl17 shows predominantly female expression following the two-cell stage in mouse embryos. Nucleic acids research 38, 3672-3681.

Kono, T., Obata, Y., Wu, Q., Niwa, K., Ono, Y., Yamamoto, Y., Park, E.S., Seo, J.-S., and Ogawa, H. (2004). Birth of parthenogenetic mice that can develop to adulthood. Nature 428, 860.

Kono, T., Obata, Y., Yoshimzu, T., Nakahara, T., and Carroll, J. (1996). Epigenetic modifications during oocyte growth correlates with extended parthenogenetic development in the mouse. Nature genetics 13 , 91.

Kono, T., Sotomaru, Y., Katsuzawa, Y., and Dandolo, L. (2002). Mouse parthenogenetic embryos with monoallelic H19 expression can develop to day 17.5 of gestation. Developmental biology 243, 294300 .

Leighton, P.A., Ingram, R.S., Eggenschwiler, J., Efstratiadis, A., and Tilghman, S.M. (1995).

Disruption of imprinting caused by deletion of the $\mathrm{H} 19$ gene region in mice. Nature 375, 34.

Li, Q., Li, Y., Yang, S., Huang, S., Yan, M., Ding, Y., Tang, W., Lou, X., Yin, Q., Sun, Z., et al. (2018). CRISPR-Cas9-mediated base-editing screening in mice identifies DND1 amino acids that are critical for primordial germ cell development. Nature cell biology 20, 1315-1325.

Li, W., Shuai, L., Wan, H., Dong, M., Wang, M., Sang, L., Feng, C., Luo, G.Z., Li, T., Li, X., et al. (2012). Androgenetic haploid embryonic stem cells produce live transgenic mice. Nature 490, 407-413. Li, Y., and Li, J. (2019). Technical advances contribute to the study of genomic imprinting. PLoS genetics 15 , e1008151.

Li, Z., Wan, H., Feng, G., Wang, L., He, Z., Wang, Y., Wang, X.J., Li, W., Zhou, Q., and Hu, B. (2016). Birth of fertile bimaternal offspring following intracytoplasmic injection of parthenogenetic haploid embryonic stem cells. Cell research 26, 135-138.

Lin, S.P., Youngson, N., Takada, S., Seitz, H., Reik, W., Paulsen, M., Cavaille, J., and Ferguson-Smith, A.C. (2003). Asymmetric regulation of imprinting on the maternal and paternal chromosomes at the Dlk1-Gt12 imprinted cluster on mouse chromosome 12. Nature genetics 35, 97-102.

Liu, W., Liu, X., Wang, C., Gao, Y., Gao, R., Kou, X., Zhao, Y., Li, J., Wu, Y., Xiu, W., et al. (2016). Identification of key factors conquering developmental arrest of somatic cell cloned embryos by combining embryo biopsy and single-cell sequencing. Cell Discov 2, 1-15.

Lopez, M.F., Dikkes, P., Zurakowski, D., and Villa-Komaroff, L. (1996). Insulin-like growth factor II affects the appearance and glycogen content of glycogen cells in the murine placenta. Endocrinology $137,2100-2108$.

McGrath, J., and Solter, D. (1984). Completion of mouse embryogenesis requires both the maternal and paternal genomes. Cell 37, 179-183. 
Morrione, A., Valentinis, B., Xu, S.-q., Yumet, G., Louvi, A., Efstratiadis, A., and Baserga, R. (1997). Insulin-like growth factor II stimulates cell proliferation through the insulin receptor. Proceedings of the National Academy of Sciences 94, 3777.

Picelli, S., Faridani, O.R., Bjorklund, A.K., Winberg, G., Sagasser, S., and Sandberg, R. (2014). Fulllength RNA-seq from single cells using Smart-seq2. Nature protocols 9, 171-181.

Rossant, J., and Cross, J.C. (2001). Placental development: lessons from mouse mutants. Nature reviews Genetics 2, 538-548.

Schmidt, J.V., Matteson, P.G., Jones, B.K., Guan, X.J., and Tilghman, S.M. (2000). The Dlk1 and Gt12 genes are linked and reciprocally imprinted. Genes Dev 14, 1997-2002.

Sibley, C.P., Coan, P.M., Ferguson-Smith, A.C., Dean, W., Hughes, J., Smith, P., Reik, W., Burton, G.J., Fowden, A.L., and Constancia, M. (2004). Placental-specific insulin-like growth factor 2 (Igf2) regulates the diffusional exchange characteristics of the mouse placenta. Proceedings of the National Academy of Sciences of the United States of America 101, 8204-8208.

Sun, M., Maliqueo, M., Benrick, A., Johansson, J., Shao, R., Hou, L., Jansson, T., Wu, X., and StenerVictorin, E. (2012). Maternal androgen excess reduces placental and fetal weights, increases placental steroidogenesis, and leads to long-term health effects in their female offspring. American journal of physiology Endocrinology and metabolism 303, E1373-1385.

Surani, M.A.H., Barton, S.C., and Norris, M.L. (1984). Development of reconstituted mouse eggs suggests imprinting of the genome during gametogenesis. Nature 308, 548.

Takada, S., Paulsen, M., Tevendale, M., Tsai, C.E., Kelsey, G., Cattanach, B.M., and Ferguson-Smith, A.C. (2002). Epigenetic analysis of the Dlk1-Gt12 imprinted domain on mouse chromosome 12: implications for imprinting control from comparison with Igf2-H19. Hum Mol Genet 11, 77-86. Takada, S., Tevendale, M., Baker, J., Georgiades, P., Campbell, E., Freeman, T., Johnson, M.H., Paulsen, M., and Ferguson-Smith, A.C. (2000). Delta-like and gtl2 are reciprocally expressed, differentially methylated linked imprinted genes on mouse chromosome 12. Curr Biol 10, 1135-1138. Tarkowski, A.K. (1975). Induced parthenogenesis in the mouse. Symp Soc Dev Biol, 107-129. Thorvaldsen, J.L., Duran, K.L., and Bartolomei, M.S. (1998). Deletion of the H19 differentially methylated domain results in loss of imprinted expression of H19 and Igf2. Genes \& Development 12, 3693-3702.

Thorvaldsen, J.L., Mann, M.R., Nwoko, O., Duran, K.L., and Bartolomei, M.S. (2002). Analysis of sequence upstream of the endogenous H19 gene reveals elements both essential and dispensable for imprinting. Molecular and cellular biology 22, 2450-2462.

Tremblay, K.D., Duran, K.L., and Bartolomei, M.S. (1997). A 5' 2-kilobase-pair region of the imprinted mouse H19 gene exhibits exclusive paternal methylation throughout development. Molecular and Cellular Biology 17, 4322-4329.

Wang, L., and Li, J. (2019). "Artificial spermatid"-mediated genome editing. Biol Reprod.

Wei, L., Wang, X., Yang, S., Yuan, W., and Li, J. (2017). Efficient generation of the mouse model with a defined point mutation through haploid cell-mediated gene editing. J Genet Genomics 44, 461-463.

Wu, Y., Liang, D., Wang, Y., Bai, M., Tang, W., Bao, S., Yan, Z., Li, D., and Li, J. (2013). Correction of a genetic disease in mouse via use of CRISPR-Cas9. Cell Stem Cell 13, 659-662.

Yagi, M., Kishigami, S., Tanaka, A., Semi, K., Mizutani, E., Wakayama, S., Wakayama, T., Yamamoto, T., and Yamada, Y. (2017). Derivation of ground-state female ES cells maintaining gamete-derived DNA methylation. Nature 548, 224-227.

Yang, H., Shi, L., Wang, B.A., Liang, D., Zhong, C., Liu, W., Nie, Y., Liu, J., Zhao, J., Gao, X., et al. 
(2012). Generation of genetically modified mice by oocyte injection of androgenetic haploid embryonic stem cells. Cell 149, 605-617.

Zhang, Y., Xiang, Y., Yin, Q., Du, Z., Peng, X., Wang, Q., Fidalgo, M., Xia, W., Li, Y., Zhao, Z.A., et al. (2018). Dynamic epigenomic landscapes during early lineage specification in mouse embryos. Nature genetics 50, 96-105.

Zhong, C., Xie, Z., Yin, Q., Dong, R., Yang, S., Wu, Y., Yang, L., and Li, J. (2016). Parthenogenetic haploid embryonic stem cells efficiently support mouse generation by oocyte injection. Cell research 26, 131-134.

Zhong, C., Yin, Q., Xie, Z., Bai, M., Dong, R., Tang, W., Xing, Y.H., Zhang, H., Yang, S., Chen, L.L., et al. (2015). CRISPR-Cas9-Mediated Genetic Screening in Mice with Haploid Embryonic Stem Cells Carrying a Guide RNA Library. Cell stem cell 17, 221-232. 